\title{
On a Cotriple Homology in a Fibred Category*
}

\author{
By \\ Hiroshi Uehara and Frank Brenneman ${ }^{\dagger}$
}

The main purpose of this paper is, among other things, to study cotriple (co) homology defined on a fibred category, which includes a unified account of introducing products of various derived functors, known or unknown, in a categorical setting. This approach is motivated by an attempt to find a suitable way, in relative homological algebra, of discussing the derived functors of a functor of two variables. In fact, this is done in this paper by considering cotriple (co) homology defined on a fibred product which is a subcategory of a product category. More precisely speaking, we introduce first a category $\mathscr{F}_{\theta}=(\mathfrak{Y}), \mathfrak{A}$, $Q)(\mathfrak{X}, \mathfrak{B}, P)$ of fibred functors $(T, \theta):(\mathfrak{X}, \mathfrak{B}, P) \rightarrow(\mathfrak{Y}), \mathfrak{A}, Q)$, which inherits the fibrewise properties of $(\mathfrak{V}, \mathfrak{A}, Q)$. Since a cotriple on the fibred category $\left(\mathfrak{X}, \mathfrak{B}, P\right.$ ) induces a cotriple on the category $\mathscr{F}_{\theta}$ in the usual sense, relative homological algebra can be applied to $\mathscr{F}_{\theta}$. Consider the situation where a fibred functor $(T, \theta)$ is defined on a fibred category $(\mathfrak{X}, \mathfrak{B}, P)$ into an abelian category $(\mathfrak{Y}, \mathfrak{X}, Q)$ and a cotriple $(G, \varepsilon, \Delta)$ is given on $(\mathfrak{X}, \mathfrak{B}, P)$. Then the cotriple (co) homology $H_{*}(\boldsymbol{T} \boldsymbol{G})$ can be defined as an object in $\mathscr{F}_{\theta}$. Moreover, if the fibred categories are both multiplicative and if the functors $G, T$ satisfy certain conditions involved in the multiplicative functors, then an external product can be defined on $H_{*}(\boldsymbol{T} \boldsymbol{G})$. For applications, $T$ is

Received December 18, 1967.

Communicated by N. Shimada.

* This research was supported in part by the National Science Foundation' and in part by the Research Foundation, Oklahoma State University.

$\dagger$ The authors are grateful to Professor Nobuo Shimada for stimulating ideas which they received while he was a Visiting Professor at the Oklahoma State University. 
a bifunctor defined on a fibred product $(\mathfrak{X} \Delta \mathfrak{X}, \mathfrak{B}, P \Delta P$ ) of a multiplicative fibred category $(\mathfrak{X}, \mathfrak{B}, P)$. As is usually the case, the EilenbergZilber theorem plays a crucial rôle in defining products. In view of the fact that a cotriple (co)homology is a derived functor it can be shown that the theorem is essentially a comparison theorem in relative homological algebra.

The notions of a cotriple on a fibred category and of induced fibred categories are introduced in the first two sections. In section 3, a category of fibred functors is discussed in order to generalize some of our previous results [6], to the case of fibred categories. The EilenbergZilber theorem is considered in section 4 . In section 5 an external product is introduced by using the previous sections. The last section is concerned with products in derived functors; Cotorsion, Coextension as well as Torsion and Extension.

\section{Cotriple on a Fibred Category}

Let $P: \mathfrak{X} \rightarrow \mathfrak{B}$ be a covariant functor of a category $\mathfrak{X}$ onto a category $\mathfrak{B}$. By the fibre $\mathfrak{X}_{B}$ over $B$ in $\mathfrak{B}$ we mean a subcategory of $\mathfrak{X}$ consisting of objects $X$ in $\mathfrak{X}$ such that $P(X)=B$ and of morphisms $f$ in $\mathfrak{X}$ such that $P(f)=1_{B}$. The inclusion functor of $\mathfrak{X}_{B}$ into $\mathfrak{X}$ is denoted by $J_{B}: \mathfrak{X}_{B} \rightarrow \mathfrak{X}$.

Definition 1.1. A fibred category of pull back type (push out type $)$ is a triple $(\mathfrak{X}, \mathfrak{B}, P)$ such that $P: \mathfrak{X} \rightarrow \mathfrak{B}$ is a functor of a category $\mathfrak{X}$ onto a category $\mathfrak{B}$, satisfying the following conditions:

1) For each morphism $\alpha: B \rightarrow B^{\prime}$ in $\mathfrak{B}$ there is associated a functor $\alpha^{*}: \mathfrak{X}_{B^{\prime}} \rightarrow \mathfrak{X}_{B}\left(\alpha_{*}: \mathfrak{X}_{B} \rightarrow \mathfrak{X}_{B^{\prime}}\right)$ and a natural transformation $\xi_{\alpha}: J_{B^{\circ}} \alpha^{*} \rightarrow J_{B^{\prime}}\left(\xi_{\alpha}\right.$ : $\left.J_{B} \rightarrow J_{B^{\prime}} \circ \alpha_{*}\right)$.

2) For each $B$ in $\mathfrak{B}, 1_{B}^{*}=I \mathfrak{x}_{B}\left(1_{B} *=I_{\mathfrak{x}_{B}}\right)$.

3) For $\alpha: B \rightarrow B^{\prime}$ and $\beta: B^{\prime} \rightarrow B^{\prime \prime}$,

$$
\xi_{\beta \alpha}=\xi_{\beta} \circ \xi_{\alpha} \beta^{*} \quad\left(\xi_{\beta \alpha}=\xi_{\beta} \alpha_{*} \circ \xi_{\alpha}\right)
$$

4) For each $f: X \rightarrow X^{\prime}$ in $\mathfrak{X}$ with $P(f)=\alpha: B \rightarrow B^{\prime}$ there exists a unique morphism $f^{\prime}: X \rightarrow \alpha^{*}\left(X^{\prime}\right)\left(f^{\prime}: \alpha_{*}(X) \rightarrow X^{\prime}\right)$ in $\mathfrak{X}_{B}$ (in $\mathfrak{X}_{B^{\prime}}$ ) such that 


$$
f=\xi_{\alpha}\left(X^{\prime}\right) \circ f^{\prime} \quad\left(f=f^{\prime} \circ \xi_{\alpha}(X)\right) .
$$

From the definition it is immediate to see that for $\alpha: B \rightarrow B^{\prime}$ and $\beta: B^{\prime} \rightarrow B^{\prime \prime},(\beta \alpha)^{*}=\alpha^{*} \beta^{*}\left((\beta \alpha)_{*}=\beta_{*} \alpha_{*}\right)$.

Given a fibred category $(\mathfrak{X}, \mathfrak{B}, P$, ) of pull back type. Let $\hat{\mathfrak{X}}$ and $\widehat{\mathfrak{B}}$ be opposite categories of $\mathfrak{X}$ and $\mathfrak{B}$ respectively, and let $\widehat{P}: \widehat{\mathfrak{X}} \rightarrow \widehat{\mathfrak{B}}$ be a covariant functor defined by $\widehat{P}(\widehat{X})=\widehat{P(X)}$ and $\widehat{P}(\hat{f})=\widehat{P(f)}$ for each object $X$ and each morphism $f$ in $\mathfrak{X}$. Defining a functor $\alpha_{*}: \hat{\mathfrak{X}}_{\widehat{B}^{\prime}}$ $\rightarrow \widehat{\mathrm{X}}_{\widehat{B}}$ by $\alpha_{*}\left(\widehat{X}^{\prime}\right)=\widehat{\alpha^{*}\left(X^{\prime}\right)}$ and $\alpha_{*}(\hat{g})=\widehat{\alpha^{*}(g)}$ for a morphism $\alpha: B \rightarrow$ $B^{\prime}$ in $\mathfrak{B}$, an object $X^{\prime}$ in $\mathfrak{X}_{B^{\prime}}$, and a morphism $g$ in $\mathfrak{x}_{B^{\prime}}$, and defining a natural transformation $\xi_{\widehat{\alpha}}: J_{\widehat{B}^{\prime}} \rightarrow J_{\widehat{B}}^{\circ} \alpha_{*}$ by $\xi_{\widehat{\alpha}}\left(\widehat{X}^{\prime}\right)=\hat{\xi}_{\alpha}\left(X^{\prime}\right)$, we have a fibred category $(\widehat{\mathfrak{X}}, \widehat{\mathfrak{B}}, \widehat{P})$ of push out type, which is called the opposite fibred category of $(\mathfrak{X}, \mathfrak{B}, P)$. This observation shows that the opposite fibred category is of push out type (of pull back type), if a given fibred category is of pull back type (of push out type). Henceforth all fibred categories will be considered to be of pull back type, unless otherwise stated, because the reader can formulate the corresponding definitions, propositions, and theorems for those of push out type by passing to the opposite fibred category.

Definition 1.2. Given fibred categories $(\mathfrak{X}, \mathfrak{B}, P)$ and $(\mathfrak{Y}, \mathfrak{C}, Q)$. A pair of functors $(F, \theta), F: \mathfrak{X} \rightarrow \mathfrak{Y})$ and $\theta: \mathfrak{B} \rightarrow(\mathfrak{C}$, is called a functor of fibred categories (fibred functor) if $Q \circ F=\theta \circ P$.

By definition it is immediate to see that for each $B$ in $\mathfrak{B}$ the functor $(F, \theta)$ induces a functor $F_{B}: \mathfrak{X}_{B} \rightarrow \bigvee_{\theta(B)}$.

Proposition 1.3. Given a functor $(F, \theta):(\mathfrak{X}, \mathfrak{B}, P) \rightarrow(\mathfrak{Y}), \mathfrak{S}, Q)$ of fibred categories. Then, for each $\alpha: B \rightarrow B^{\prime}$ in $\mathfrak{B}$ there exists a natural transformation $\eta_{\alpha}: F_{B} \circ \alpha^{*} \rightarrow \theta(\alpha) * \circ F_{B^{\prime}}$ satisfying the following conditions; for $\alpha: B \rightarrow B^{\prime}$ and $\beta: B^{\prime} \rightarrow B^{\prime \prime}$ in $\mathfrak{B}$,

$$
\eta_{\beta \alpha}=\left(\theta(\alpha) * \eta_{\beta}\right) \circ\left(\eta_{\alpha} \beta^{*}\right)
$$

Proof. From the axiom 4 of Definition 1.1 it is seen that for $\alpha: B \rightarrow B^{\prime}$ in $\mathfrak{B}$ and for an object $X^{\prime}$ in $\mathfrak{X}_{B^{\prime}}$ there exists a unique morphism in $\mathfrak{Y}_{\theta(B)}$

$$
\left(F\left(\xi_{\alpha}\left(X^{\prime}\right)\right)\right)^{\prime}: F_{B}\left(\alpha^{*}\left(X^{\prime}\right)\right) \rightarrow \theta(\alpha)^{*}\left(F_{B^{\prime}}\left(X^{\prime}\right)\right)
$$


satisfying

$$
\xi_{\theta(\alpha)}\left(F_{B^{\prime}}\left(X^{\prime}\right)\right) \circ\left(F\left(\xi_{\alpha}\left(X^{\prime}\right)\right)\right)^{\prime}=F \xi_{\alpha}\left(X^{\prime}\right) .
$$

Define $\eta_{\alpha}\left(X^{\prime}\right)=\left(F\left(\xi_{\alpha}\left(X^{\prime}\right)\right)\right)^{\prime}$ for each $X^{\prime}$ in $\mathfrak{X}_{B^{\prime}}$, then $\eta_{\alpha}: F_{B} \alpha^{*} \rightarrow \theta(\alpha) * F_{B^{\prime}}$ is a natural transformation.

By definition of $\eta_{\beta \alpha}$,

$$
F\left(\xi_{\beta \alpha}\left(X^{\prime \prime}\right)\right)=\xi_{\theta\left(\alpha^{*}\right)}\left(F_{B^{\prime \prime}}\left(X^{\prime \prime}\right)\right) \circ \eta_{\beta \alpha}\left(X^{\prime \prime}\right)
$$

for an object $X^{\prime \prime}$ in $\mathfrak{X}_{B^{\prime \prime}}$ and morphisms $\alpha: B \rightarrow B^{\prime}, \beta: B^{\prime} \rightarrow B^{\prime \prime}$ in $\mathfrak{B}$. From the axiom 3 of 1.1 and from the definition of $\eta_{\alpha}, \eta_{\beta}$,

$$
\begin{aligned}
F\left(\xi_{\beta \alpha}\left(X^{\prime \prime}\right)\right) & =F\left(\xi_{\beta}\left(X^{\prime \prime}\right) \circ \xi_{\alpha}\left(\beta *\left(X^{\prime \prime}\right)\right)\right) \\
& =F\left(\xi_{\beta}\left(X^{\prime \prime}\right)\right) \circ F\left(\xi_{\alpha}\left(\beta *\left(X^{\prime \prime}\right)\right)\right) \\
& =\xi_{\theta(\beta)}\left(F_{B^{\prime \prime}}\left(X^{\prime \prime}\right)\right) \circ \eta_{\beta}\left(X^{\prime \prime}\right) \circ \xi_{\theta(\alpha)}\left(F_{B^{\prime}}\left(\beta^{*}\left(X^{\prime \prime}\right)\right)\right) \circ \eta_{\alpha}\left(\beta^{*}\left(X^{\prime \prime}\right)\right) .
\end{aligned}
$$

Since $\xi_{\theta(\alpha)}$ in a natural transformation,

$$
\eta_{\beta}\left(X^{\prime \prime}\right) \circ \xi_{\theta(\alpha)}\left(F_{B^{\prime}}\left(\beta^{*}\left(X^{\prime \prime}\right)\right)\right)=\xi_{\theta(\alpha)}\left(\theta(\beta) *\left(F_{B^{\prime \prime}}\left(X^{\prime \prime}\right)\right)\right) \circ \theta(\alpha) *\left(\eta_{\beta}\left(X^{\prime \prime}\right)\right) .
$$

Hence

$$
\begin{aligned}
F\left(\xi_{\beta \alpha}\left(X^{\prime \prime}\right)\right)= & \xi_{\theta(\beta)}\left(F_{B^{\prime \prime}}\left(X^{\prime \prime}\right)\right) \circ \xi_{\theta(\alpha)}\left(\theta(\beta)^{*}\left(F_{B^{\prime \prime}}\left(X^{\prime \prime}\right)\right)\right) \\
& \circ \theta(\alpha) *\left(\eta_{\beta}\left(X^{\prime \prime}\right)\right) \circ \eta_{\alpha}\left(\beta^{*}\left(X^{\prime \prime}\right)\right) \\
= & \xi_{\theta(\beta \alpha)}\left(F_{B^{\prime \prime}}\left(X^{\prime \prime}\right)\right) \circ \theta(\alpha) *\left(\eta_{\beta}\left(X^{\prime \prime}\right)\right) \circ \eta_{\alpha}\left(\beta^{*}\left(X^{\prime \prime}\right)\right) .
\end{aligned}
$$

Comparing (1) with (2), we obtain

$$
\eta_{\beta \alpha}\left(X^{\prime \prime}\right)=\theta(\alpha) *\left(\eta_{\beta}\left(X^{\prime \prime}\right)\right) \circ \eta_{\alpha}\left(\beta\left(X^{\prime \prime}\right)\right)
$$

by the uniqueness of a component of the morphism $F\left(\xi_{\beta \alpha}\left(X^{\prime \prime}\right)\right)$. Therefore, the proof is completed.

Definition 1.4. By a cotriple $(G, \varepsilon, \Delta)$ on a fibred category (X, $\mathfrak{B}, P)$ we mean a cotriple $(G, \varepsilon, \Delta)$ on the category $\mathfrak{X}$ such that a pair of functors $\left(G, I_{\mathfrak{B}}\right)$ with the identity functor $I_{\mathfrak{B}}$, is a functor $_{\mathfrak{\imath}}$ of the fibred category into itself, satisfying the conditions

$$
P \varepsilon(X)=P \Delta(X)=1_{P(X)},
$$

for each object $X$ in $\mathfrak{X}$.

Given a cotriple $(G, \varepsilon, \Delta)$ on a fibred category $(\mathfrak{X}, \mathfrak{B}, P)$ of pull back type. Considering the opposite fibred category $(\hat{X}, \widehat{\mathfrak{B}}, \widehat{P})$ of push 
out type and defining

$$
\widehat{G}(\widehat{X})=\widehat{G(X)}, \widehat{G}(\hat{f})=\widehat{G(f)}, \widehat{\varepsilon}(\widehat{X})=\widehat{\varepsilon(X)} \text {, and } \hat{\Delta}(\widehat{X})=\widehat{\Delta(X)}
$$

for each object $X$ in $X$ and a morphism $f$ in $\varkappa$, we have a functor $(\widehat{G}$, $I \hat{\mathfrak{B}})$ of the opposite fibred category such that $(\widehat{G}, \hat{\varepsilon}, \hat{\Delta})$ forms a triple on $\widehat{\mathfrak{X}}$ satisfying

$$
\widehat{P}_{\widehat{\varepsilon}}(\widehat{X})=\widehat{P} \hat{\Delta}(\widehat{X})=1_{\widehat{P}(\widehat{X})}
$$

for each object $\widehat{X}$ in $\widehat{x}$.

For the sake of completeness we give

Definition 1.5. A triple $(G, \varepsilon, \Delta)$ on a category $\mathfrak{X}$ is called a triple on a fibred category $(\mathfrak{X}, P, \mathfrak{B})$ of push out type iff $\left(G, I_{\mathscr{B}}\right)$ is a functor of the fibred category into itself, satisfying

$$
P \varepsilon(X)=P \Delta(X)=1_{P(X)}
$$

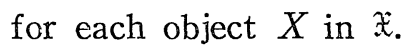

It is clear from the remark made above that if a cotriple (triple) is given on a fibred category of pull back type (push out type), then a triple (cotriple) is obtained on the opposite fibred category.

Theorem 1.6. Given a cotriple $(G, \varepsilon, \Delta)$ on a fibred category ( $\mathfrak{X}$, $\mathfrak{B}, P)$. Then the cotriple induces a cotriple $\left(G_{B}, \varepsilon_{B}, \Delta_{B}\right)$ on each fibre $\mathfrak{X}_{B}$ such that

1) for morphisms $\alpha: B \rightarrow B^{\prime}, \beta: B^{\prime} \rightarrow B^{\prime \prime}$ there exists a natural transformation $\eta_{\alpha}: G_{B} \alpha^{*} \rightarrow \alpha^{*} G_{B^{\prime}}$ satisfying the conditions that

$$
\eta_{\beta \alpha}=\left(a^{*} \eta_{\beta}\right) \circ\left(\eta_{\alpha} \beta^{*}\right) \text { and } \eta_{1_{B}}(X)=1_{X} \text { for } X \text { with } P(X)=B \text {, }
$$

2) $\varepsilon_{B} \alpha^{*}=\alpha^{*} \varepsilon_{B^{\prime}} \circ \eta_{\alpha}$ for $\alpha: B \rightarrow B^{\prime}$.

Conversely, if a cotriple $\left(G_{B}, \varepsilon_{B}, \Delta_{B}\right)$ is given on each fibre $\mathfrak{X}_{B}$ of a fibred category $(\mathfrak{X}, \mathfrak{B}, P)$ in such a way that the two conditions stated above are satisfied, then there exists a cotriple $(G, \varepsilon, \Delta)$ on the fibred category which induces the cotriple $\left(G_{B}, \varepsilon_{B}, \Delta_{B}\right)$ on each fibre $\mathfrak{X}_{B}$.

Proof. Assume that a cotriple is given on a fibred category. Since $\left(G, I_{\mathfrak{B}}\right)$ is a functor of the fibred category, it induces a functor $G_{B}: \mathfrak{X}_{B} \rightarrow \mathfrak{X}_{B}$ for each $B$ in $\mathfrak{B}$, and there exists a natural transformation 
$\eta_{\alpha}: G_{B} \alpha^{*} \rightarrow \alpha^{*} G_{B^{\prime}}$ for $\alpha: B \rightarrow B^{\prime}$ satisfying the condition 1 ) because of proposition 1.3. Defining $\varepsilon_{B}(X)=\varepsilon(X), \Delta_{B}(X)=\Delta(X)$ for $X$ with $P(X)=B$, we have a cotriple $\left(G_{B}, \varepsilon_{B}, \Delta_{B}\right)$ on $\mathfrak{x}_{B}$. Consider a diagram

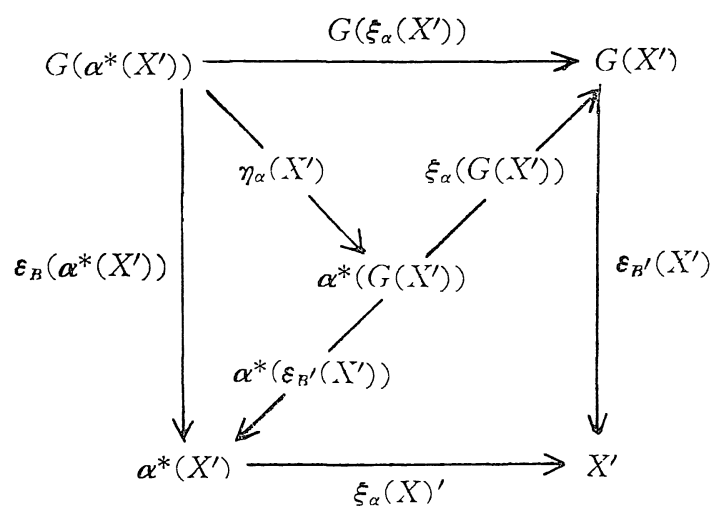

Since $\varepsilon$ is a natural transformation, $\left.\xi_{\alpha}\left(X^{\prime}\right) \circ \varepsilon_{B}\left(\alpha^{*}\left(X^{\prime}\right)\right)=\varepsilon_{B^{\prime}}\left(X^{\prime}\right)\right) \circ$ $G\left(\xi_{\alpha}\left(X^{\prime}\right)\right)$. By definition of $\eta_{\alpha}, G\left(\xi_{\alpha}\left(X^{\prime}\right)\right)=\xi_{\alpha}\left(G\left(X^{\prime}\right)\right) \circ \eta_{\alpha}\left(X^{\prime}\right)$. Since $\xi_{\alpha}$ is a natural transformation, $\varepsilon_{B^{\prime}}\left(X^{\prime}\right) \circ \xi_{\alpha}\left(G\left(X^{\prime}\right)\right)=\xi_{\alpha}\left(X^{\prime}\right) \circ \alpha^{*}\left(\varepsilon_{B^{\prime}}\left(X^{\prime}\right)\right)$. Hence, by a diagram chasing, $\xi_{\alpha}\left(X^{\prime}\right) \circ \varepsilon_{B}\left(\alpha^{*}\left(X^{\prime}\right)\right)=\xi_{\alpha}\left(X^{\prime}\right) \circ \alpha^{*}\left(\varepsilon_{B^{\prime}}\left(X^{\prime}\right)\right) \circ$ $\eta_{\alpha}\left(X^{\prime}\right)=\varphi$. By the uniqueness of a component of the morphism $\varphi$, we have

$$
\varepsilon_{B}\left(\alpha^{*}\left(X^{\prime}\right)\right)=\alpha^{*}\left(\varepsilon\left(X^{\prime}\right)\right) \circ \eta_{\alpha}\left(X^{\prime}\right)
$$

for each object $X^{\prime}$ in $\mathfrak{x}_{B^{\prime}}$. Therefore the first half of the theorem has been proved.

First let us define a functor $G: \mathfrak{X} \rightarrow \mathfrak{X}$ in terms of a cotriple given on each fibre. For an object $X$ with $P(X)=B$ define $G(X)=G_{B}(X)$. For a morphism $f: X \rightarrow X^{\prime}$ with $P(f)=\alpha: B \rightarrow B^{\prime}$ consider the diagram

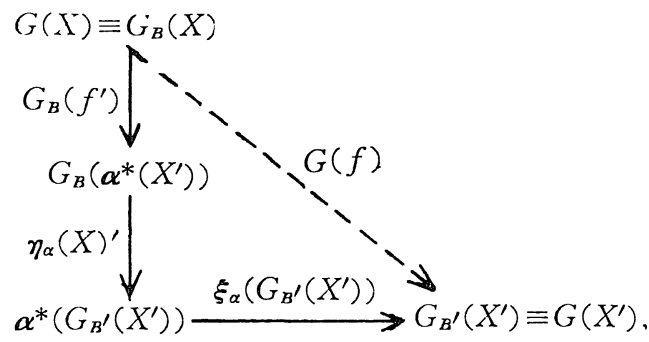


where $f=\xi_{\alpha}\left(X^{\prime}\right) \circ f^{\prime}$, and define $G(f)=\xi_{\alpha}\left(G_{B^{\prime}}\left(X^{\prime}\right)\right) \circ \eta_{\alpha}\left(X^{\prime}\right) \circ G_{B}\left(f^{\prime}\right)$. Note that if $f$ is in $\mathfrak{x}_{B}$, then $G(f)=G_{B}(f)$. Hence $G\left(1_{X}\right)=1_{G(X)}$. For morphisms $f: X \rightarrow X^{\prime}, g: X^{\prime} \rightarrow X^{\prime \prime}$ with $P(f)=\alpha: B \rightarrow B^{\prime}, P(g)=\beta: B^{\prime}$ $\rightarrow B^{\prime \prime}$, consider the diagram

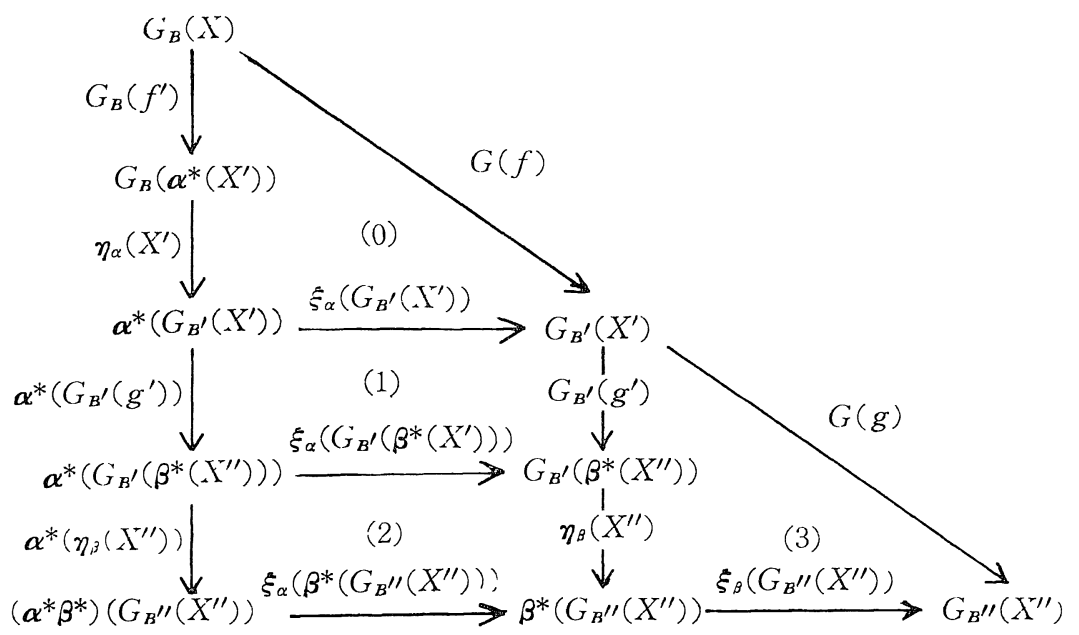

then the triangles (0), (3) are commutative by definition of $G(f)$ and $G(g)$, and the rectangles (1) and (2) are commutative because $\xi_{\alpha}$ is a natural transformation.

Hence $G(g) \circ G(f)=\xi_{\beta}\left(G_{B^{\prime \prime}}\left(X^{\prime \prime}\right)\right) \circ \xi_{\alpha}\left(\beta^{*}\left(G_{B^{\prime \prime}}\left(X^{\prime \prime}\right)\right)\right) \circ \alpha^{*}\left(\eta_{\beta}\left(X^{\prime \prime}\right)\right) \circ$ $\alpha^{*}\left(G_{B^{\prime}}\left(g^{\prime}\right)\right) \circ \eta_{\alpha}\left(X^{\prime}\right) \circ G_{B}\left(f^{\prime}\right)$ by a diagram chasing. Since $\eta_{\alpha}$ is a natural transformation, $\alpha^{*}\left(G_{B^{\prime}}\left(g^{\prime}\right)\right) \circ \eta_{\alpha}\left(X^{\prime}\right)=\eta_{\alpha}\left(\beta^{*}\left(X^{\prime}\right)\right) \circ G_{B}\left(\alpha^{*}\left(g^{\prime}\right)\right)$. Also we have $G_{B}\left((g \circ f)^{\prime}\right)=G_{B}\left(\alpha^{*}\left(g^{\prime}\right)\right) \circ G_{B}\left(f^{\prime}\right)$, so that

$$
\begin{aligned}
G(g) \circ G(f)= & \xi_{\beta}\left(G_{B^{\prime \prime}}\left(X^{\prime \prime}\right)\right) \circ \xi_{\alpha}\left(\beta^{*} G_{B^{\prime \prime}}\left(X^{\prime \prime}\right)\right) \circ \alpha^{*}\left(\eta_{\beta}\left(X^{\prime \prime}\right)\right) \\
& \circ \eta_{\alpha}\left(\beta^{*}\left(X^{\prime}\right)\right) \circ G_{B}\left(\alpha^{*}\left(g^{\prime}\right)\right) \circ G_{B}\left(f^{\prime}\right) \\
= & \xi_{\beta_{\alpha}}\left(G_{B^{\prime \prime}}\left(X^{\prime \prime}\right)\right) \circ \eta_{\beta \alpha}\left(X^{\prime \prime}\right) \circ G_{B}\left((g \circ f)^{\prime}\right) \\
= & G(g \circ f) .
\end{aligned}
$$

From the construction it is easily seen that $\left(G, I_{\mathfrak{B}}\right)$ is a functor of fibred categories.

Define $\varepsilon: G \rightarrow I_{\mathfrak{X}}$ by $\varepsilon(X)=\varepsilon_{B}(X)$ for $X$ with $P(X)=B$. Then $\varepsilon$ is a natural transformation. To see this consider the diagram for $f$ : $X \rightarrow X^{\prime}$ with $P(f)=\alpha: B \rightarrow B^{\prime}$. 


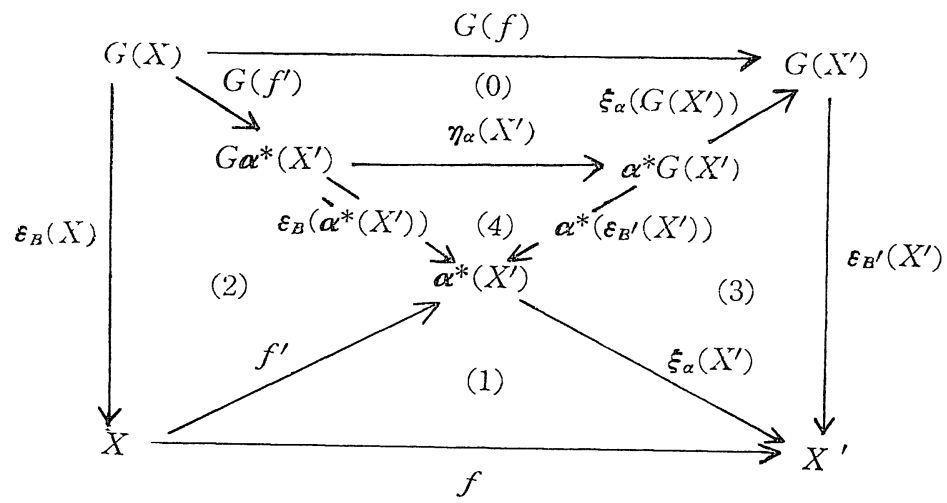

By definition of $G(f),(0)$ is commutative, (1) is commutative from the axiom 4 of 1.1 , (2) and (3) are commutative because $\varepsilon_{B}$ and $\varepsilon_{B^{\prime}}$ are natural transformations, and (4) is commutative by assumption. By a diagram chasing we have

$$
f \circ \varepsilon(X)=\varepsilon\left(X^{\prime}\right) \circ G(f) .
$$

Define $\Delta: G \rightarrow G^{2}$ by $\Delta(X)=\Delta_{B}(X)$ for $X$ with $P(X)=B$. For a morphism $f: X \rightarrow X^{\prime}$ with $P(f)=\alpha: B \rightarrow B^{\prime}$ consider the diagram

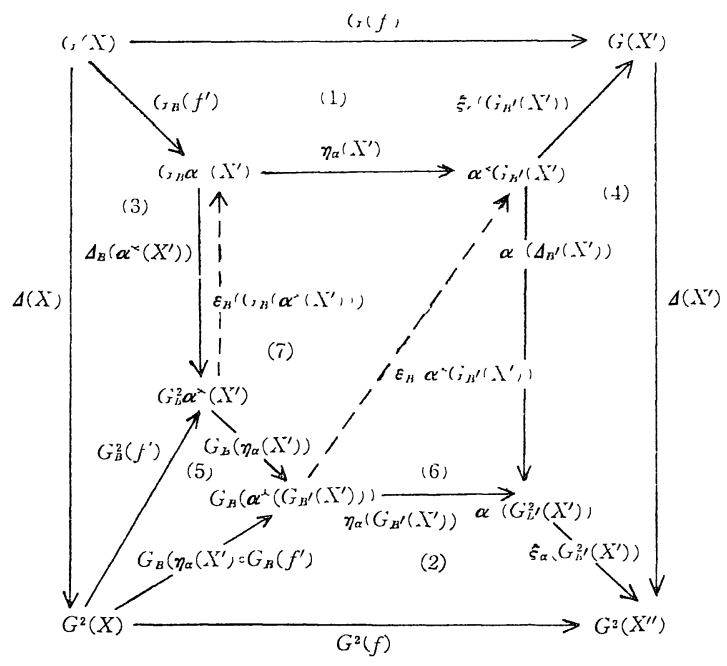

then the commutativities of (1), (2), (3), (4), (5) are obvious. The only commutativity we need to show $\Delta\left(X^{\prime}\right) \circ G(f)=G^{2}(f) \circ \Delta(X)$, is $\alpha^{*}\left(\Delta_{B^{\prime}}\right.$ $\left.\left.X^{\prime}\right)\right) \circ \eta_{\alpha}\left(X^{\prime}\right)=\eta_{\alpha}\left(G_{B^{\prime}}\left(X^{\prime}\right)\right) \circ G_{B}\left(\eta_{\alpha}\left(X^{\prime}\right)\right) \circ \Delta_{B}\left(\alpha^{*}\left(X^{\prime}\right)\right)$. From the assumed 
condition 2) relating $\varepsilon_{B}$ and $\varepsilon_{B^{\prime}}$ we have $\alpha^{*}\left(\Delta_{B^{\prime}}\left(X^{\prime}\right)\right){ }^{\circ} \varepsilon_{B}\left(\alpha^{*}\left(G_{B^{\prime}}\left(X^{\prime}\right)\right)\right)$ $=\alpha^{*}\left(\Delta_{B^{\prime}}\left(X^{\prime}\right)\right) \circ \alpha^{*}\left(\varepsilon_{B^{\prime}}\left(G_{B^{\prime}}\left(X^{\prime}\right)\right) \circ \eta_{\alpha}\left(G_{B^{\prime}}\left(X^{\prime}\right)\right)=\alpha^{*}\left(\Delta_{B^{\prime}}\left(X^{\prime}\right) \circ \varepsilon_{B^{\prime}} G_{B^{\prime}}\left(X^{\prime}\right)\right) \circ\right.$ $\eta_{\alpha}\left(G_{B^{\prime}}\left(X^{\prime}\right)\right)=\eta_{\alpha}\left(G_{B^{\prime}}\left(X^{\prime}\right)\right)$, so that the commutativity of $(6)$ is proved. From the commutativity of $\left.(7), \varepsilon_{B}\left(G_{B} \alpha^{*}\left(X^{\prime}\right)\right)\right) \circ \eta_{\alpha}\left(X^{\prime}\right)=\varepsilon_{B}\left(\alpha^{*} G_{B^{\prime}}\left(X^{\prime}\right)\right)$ - $G_{B}\left(\eta_{\alpha}\left(X^{\prime}\right)\right)$ and from $\left.\Delta_{B}\left(\alpha^{*}\left(X^{\prime}\right)\right) \circ \varepsilon_{B} G_{B}\left(\alpha^{*} X^{\prime}\right)\right)=1_{G_{B}}\left(\alpha^{*}\left(X^{\prime}\right)\right)$, we obtain the desired commutativity. Since the properties for a cotriple $(G$, $\varepsilon, \Delta)$ are readily proved, the proof of the theorem has been completed.

Before we close the present section, let us consider an example of a triple of a fibred category, which will be used in later sections.

Let $\Lambda$ be an algebra over a commutative ring $K$ with unity and let $\mu: \Lambda \otimes \Lambda \rightarrow \Lambda$ and $\rho: K \rightarrow \Lambda$ be the multiplication and the identity respectively. By a $\Lambda$-contramodule $M$ we mean a $K$-module together with a $K$-homomorphism ${ }_{M} \psi: M \rightarrow \operatorname{Hom}_{K}(\Lambda, M)$ such that the diagrams
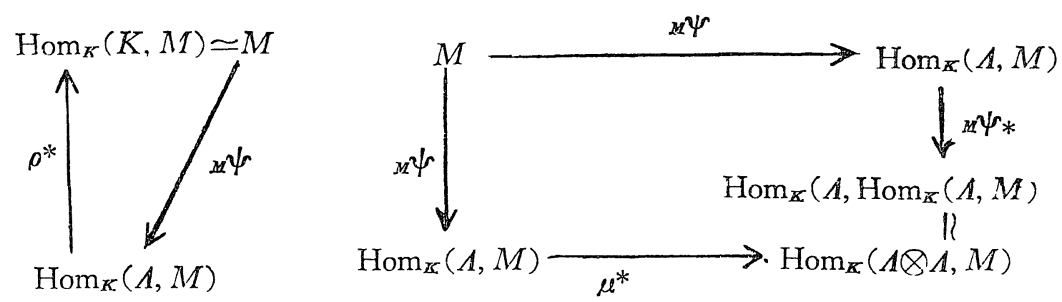

are commutative. A morphism of $\Lambda$-contramodules is a $K$-homomorphism $f: M \rightarrow M^{\prime}$ such that the diagram

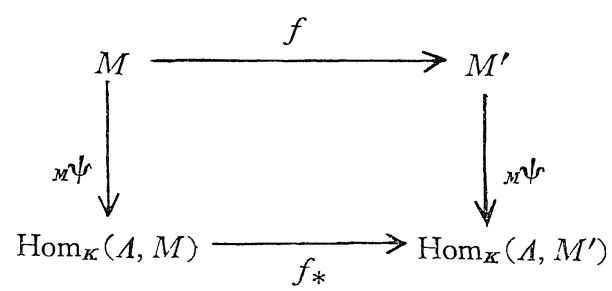

is commutative. Let $\mathfrak{M}_{A}^{\ddagger}$ denote the category of all $\Lambda$-contramodules with morphisms of $A$-contramodules and let ${ }_{K} \mathfrak{M}$ be the category of $K$-modules with $K$-homomorphisms as morphisms. Adjoint functors, $U:{ }_{A}^{\sharp} \mathfrak{M} \rightarrow{ }_{K} \mathfrak{M}$ and $S:{ }_{K} \mathfrak{M} \rightarrow{ }_{A}^{\sharp} \mathfrak{M}$ will be defined in such a manner as $U \dashv S:\left({ }_{K} \mathfrak{M},{ }_{A}{ }_{A} \mathfrak{M}\right)$, so that we have a triple $\left({ }_{1} G,{ }_{1} \varepsilon,{ }_{\Lambda} \Delta\right)$ with ${ }_{1} G=S \circ U$. Let $U$ be the forgetful functor and let $S$ be defined by $S(A)=\operatorname{Hom}_{K}(\Lambda, A)$ and $S(f)=f_{*}: \operatorname{Hom}_{K}(\Lambda, A) \rightarrow \operatorname{Hom}_{K}\left(\Lambda, A^{\prime}\right)$ for $f: A \rightarrow A^{\prime}$ in ${ }_{K} \mathfrak{M}$. Notice 
that $\operatorname{Hom}_{K}(\Lambda, A)$ is a $\Lambda$-contramodule with structure given by $\mu^{*}$ : $\operatorname{Hom}_{K}(\Lambda, A) \rightarrow \operatorname{Hom}_{K}(\Lambda \otimes \Lambda, A) \simeq \operatorname{Hom}_{K}\left(\Lambda, \operatorname{Hom}_{K}(\Lambda, A)\right)$. Then ${ }_{i} \varepsilon(M)=$ ${ }_{M} \psi$ and ${ }_{\Lambda} \Delta(M)=(1 \otimes \rho) *$.

Let $\widehat{\mathfrak{U}}$ be the opposite category of the category $\mathfrak{U}$ of all $K$-algebras with algebra homomorphisms, and let $\# \mathfrak{M}$ be the category of all ordered pairs $(\hat{\Lambda}, M)$, where $\Lambda$ is a $K$-algebra and $M$ is a $A$-contramodule, together with morphisms $(\hat{\alpha}, f):(\widehat{\Lambda}, M) \rightarrow\left(\widehat{\Lambda^{\prime}}, M^{\prime}\right)$, where $\hat{\alpha}: \widehat{\Lambda} \rightarrow \widehat{\Lambda^{\prime}}$ is in $\widehat{\mathfrak{A}}$, i. e., $\alpha: \Lambda^{\prime} \rightarrow \Lambda$ is in $\mathfrak{A}$, and $f: M \rightarrow M^{\prime}$ is a $K$-homomorphism satisfying the commutativity of the diagram

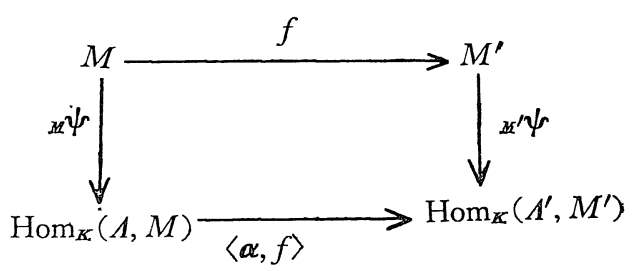

with $\langle\alpha, f\rangle(\xi)=f \circ \xi \circ \alpha$ for each $\xi \varepsilon \operatorname{Hom}_{K}(\Lambda, M)$. Composition in ${ }^{\#} \mathfrak{M}$ is defined by $(\hat{\alpha}, f) \circ(\hat{\beta}, g)=(\widehat{\beta \alpha}, f g)$. Define a covariant onto functor $P$ : ${ }^{\sharp} \mathfrak{M} \rightarrow \widehat{\mathscr{U}}$ by $P(\widehat{\Lambda}, M)=\widehat{\Lambda}$ and $P(\hat{\alpha}, f)=\hat{\alpha}$. For each $\hat{\alpha}: \widehat{\Lambda} \rightarrow \widehat{\Lambda^{\prime}}$ let $\hat{\alpha}_{*}:{ }_{\Lambda}^{*} \mathfrak{M}$ $\rightarrow \#$ 促 $M$ is given a $\Lambda^{\prime}$-contramodule structure by $M \stackrel{\lambda \sim}{\longrightarrow} \operatorname{Hom}_{K}(\Lambda, M) \stackrel{\kappa_{\alpha}}{\longrightarrow}$ $\operatorname{Hom}_{K}\left(\Lambda^{\prime}, M\right)$ with $\kappa_{\alpha}(\xi)=\xi \circ \alpha$ for each $\xi \varepsilon \operatorname{Hom}_{K}(\Lambda, M)$. Difine $\xi_{\hat{\alpha}}: J_{\widehat{A}}$ $\rightarrow I_{\widehat{\Lambda}^{\prime}} \circ \hat{\alpha}_{*}$ by $\xi_{\hat{\alpha}}(\hat{\Lambda}, M)=(\hat{\alpha}, 1):(\widehat{\Lambda}, M) \rightarrow\left(\hat{\Lambda}^{\prime}, M\right)$. Then it is straight-

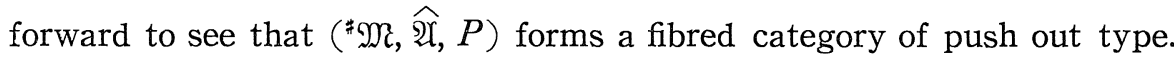

As we observed before, each fibre $\#_{A}$ over $\widehat{A}$ in $\widehat{\mathfrak{A}}$ has a triple $\left({ }_{A} G,{ }_{A} \varepsilon,{ }_{A} \Delta\right)$. If the two conditions of the dual of Theorem 1.6 are verified in this example, we have a triple $(G, \varepsilon, \Delta)$ on $(\sharp \mathfrak{M}, \widehat{\mathfrak{A}}, P)$. For $(\widehat{\Lambda}, M)$ in $\#$ Mi and for $\hat{\alpha}: \widehat{\Lambda} \rightarrow \widehat{\Lambda}^{\prime}, \hat{\alpha}_{* A} G(\widehat{\Lambda}, \widehat{M})=\left(\widehat{\Lambda}^{\prime}, \operatorname{Hom}_{K}(\Lambda, M)\right)$ and ${ }_{\Lambda^{\prime}} G \hat{\alpha}_{*}(\widehat{\Lambda}, M)=\left(\widehat{\Lambda^{\prime}}, \operatorname{Hom}_{K}\left(\Lambda^{\prime}, M\right)\right)$. Define $\eta_{\hat{\alpha}}: \hat{\alpha}_{* 1} G \rightarrow{ }_{\Lambda^{\prime}} G \hat{\alpha}_{*}$ by $\eta_{\widehat{\alpha}}(\widehat{\Lambda}, M)$ $=\left(1, \kappa_{\alpha}\right)$, where $\kappa_{\alpha}: \operatorname{Hom}_{K}(\Lambda, M) \rightarrow \operatorname{Hom}_{K}\left(\Lambda^{\prime}, M\right)$ is defined by $\kappa_{\alpha}(\xi)=$ $\xi \circ \alpha$. It is easy to see that all the necessary conditions are satisfied.

\section{Fibred Product}

Proposition 2.1 Given a fibred category $(\mathfrak{X}, \mathfrak{B}, P$ ) and a functor 
$\theta: \overline{\mathfrak{B}} \rightarrow \mathfrak{B}$. Consider a subcategory $\theta^{-1}(\mathfrak{X})$ of the product category $\mathfrak{X} \times$ $\overline{\mathfrak{B}}$ consisting of objects $(X, \bar{B})$ in $\mathfrak{X} \times \overline{\mathfrak{B}}$ with the property $P(X)=\theta(\bar{B})$ and of morphisms $(f, \bar{\alpha})$ in $\mathfrak{X} \times \overline{\mathfrak{B}}$ with $P(f)=\theta(\bar{\alpha})$. Defining $\bar{P}$ : $\theta^{-1}(\mathfrak{X}) \rightarrow \overline{\mathfrak{B}}$ by $\bar{P}(X, \bar{B})=\bar{B}, \bar{P}(f, \bar{\alpha})=\bar{\alpha}$, and $F: \theta^{-1}(\mathfrak{X}) \rightarrow \mathfrak{X}$ by $F(X, \bar{B})$ $=X, F(f, \bar{\alpha})=f$, the triple $\left(\theta^{-1}(\mathfrak{X}), \overline{\mathfrak{K}}, \bar{P}\right)$ forms a fibred category such that $(F, \theta):\left(\theta^{-1}(\mathfrak{X}), \overline{\mathfrak{B}}, \bar{P}\right) \rightarrow(\mathfrak{X}, \mathfrak{B}, P)$ is a fibred functor.

Proof. For composable morphisms $(f, \bar{\alpha}),(g, \bar{\beta})$ in $\theta^{-1}(\mathfrak{X}),(f, \bar{\alpha}) \circ$ $(g, \bar{\beta})=(f \circ g, \bar{\alpha} \circ \bar{\beta})$ is in $\theta^{-1}(\mathfrak{X})$, because $P(f \circ g)=P(f) \circ P(g)=\theta(\bar{\alpha}) \circ$ $\theta(\bar{\beta})=\theta(\bar{\alpha} \circ \bar{\beta})$. For each object $(X, \bar{B})$ in $\theta^{-1}(\mathfrak{X}),\left(1_{X}, 1_{\bar{B}}\right)$ is the identity morphism, because $P\left(1_{X}\right)=1_{P(X)}=1_{\theta(\bar{B})}$. Hence $\theta^{-1}(\mathfrak{X})$ is a subcategory of $\mathfrak{X} \times \overline{\mathfrak{B}}$. For each $\bar{\alpha}: \bar{B} \rightarrow \bar{B}^{\prime}$ define a functor $\bar{\alpha}^{*}: \theta^{-1}(\mathfrak{X})_{\bar{B}^{\prime}} \rightarrow \theta^{-1}(\mathfrak{X})_{\bar{B}}$ by $\bar{\alpha}^{*}\left(X^{\prime}, \bar{B}^{\prime}\right)=\left(\theta(\bar{\alpha})^{*}\left(X^{\prime}\right), \bar{B}\right), \bar{\alpha}^{*}\left(f, 1_{\bar{B}^{\prime}}\right)=\left(\theta(\bar{\alpha})^{*}(f), 1_{\bar{B}}\right)$ for an object $\left(X^{\prime}, \bar{B}^{\prime}\right)$ and a morphism $\left(f, 1_{\bar{B}^{\prime}}\right)$ in $\theta^{-1}(\mathfrak{X})_{\bar{B}^{\prime}}$, and define a morphism $\xi_{\bar{\alpha}}\left(X^{\prime}, \bar{B}^{\prime}\right)=\left(\xi_{\theta(\bar{\alpha})}\left(X^{\prime}\right), \bar{\alpha}\right)$. Then it is easy to see that $\left(\theta^{-1}(\mathfrak{X}), \overline{\mathfrak{B}}, \bar{P}\right)$ forms a fibred category such that $(F, \theta)$ is a fibred map. Hence the proof is completed.

Definition 2.2. $\left(\theta^{-1}(z), \overline{\mathfrak{S}}, \bar{P}\right)$ is called the fibred category induced by $\theta: \overline{\mathfrak{B}} \rightarrow \mathfrak{B}$ and the fibred category $(\ddot{X}, \mathfrak{B}, P)$. It will be denoted by $\theta^{-1}(\mathfrak{X}, \mathfrak{B}, P)$.

Definition 2.3. Given fibred categories $(\mathfrak{X}, \mathfrak{B}, P)$, (⿹ำ, $\mathfrak{B}, Q)$ and a "diagonal" functor $\Delta: \mathfrak{B} \rightarrow \mathfrak{B} \times \mathfrak{B}$ defined by $\Delta(B)=(B, B)$ and $\Delta(f)=$ $(f, f)$ for an object $B$ and a morphism $f$ in $\mathfrak{B}$. The fibred category induced by $\Delta$ and the prcduct fibred category $(\mathfrak{X} \times \mathfrak{Y}, \mathfrak{B} \times \mathfrak{B}, P \times Q)$, is called the fibred product of $(\mathfrak{X}, \mathfrak{B}, P)$ and $(\mathfrak{Y}, \mathfrak{B}, Q)$, and is denoted by $(x \Delta \mathfrak{Z}, \mathfrak{B}, P \Delta Q)$ where the product fibred category is defined in the obvious manner.

It is easy to see that the fibred product ( $\triangle \mathscr{Y}, \mathfrak{B}, P \Delta Q$ ) is a fibred category consisting of a subcategory $\mathfrak{X} \Delta \mathfrak{Y}$ of $\mathfrak{X} \times \mathfrak{Y}$ whose objects are $(X, Y)$ in $\mathfrak{X} \times \mathfrak{Y}$ with the property $P(X)=Q(Y)$ and whose morphisms are $(f, g)$ with $P(f)=Q(g)$, together with a functor $P \Delta Q: \mathfrak{X} \Delta \mathfrak{Y} \rightarrow \mathfrak{B}$ defined by $(P \Delta Q)(X, Y)=P(X)=Q(X)$ and $(P \Delta Q)(f, g)=P(f)=$ $Q(g)$. 
Definition 2.4. A fibred category $(\mathfrak{X}, \mathfrak{B}, P)$ is said to be pointed if each fibre $\mathfrak{x}_{B}$ is pointed such that for $a: B \rightarrow B^{\prime}$ in $\mathfrak{B}, \alpha^{*}: \mathfrak{X}_{B^{\prime}} \rightarrow \mathfrak{X}_{B}$ is a pointed functor.

Definition 2.5. A pointed fibred category $(\mathfrak{X}, \mathfrak{B}, P)$ is said to be preadditive if for each $\alpha$ in $\mathfrak{B}, \operatorname{hom}_{\alpha}\left(X, X^{\prime}\right)=\left\{f: X \rightarrow X^{\prime} \mid P(f)=\alpha\right\}$ is an abelian group such that $(f+g) h=f h+g h$ and $h(f+g)=h f+$ $h g$ whenever morphisms are composable.

Proposition 2.6. If $(\mathfrak{X}, \mathfrak{B}, P)$ is a preadditive fibred category, then $\mathfrak{x}_{B}$ is preadditive for each $B$ and for each $\alpha: B \rightarrow B^{\prime}$ in $\mathfrak{R}, \alpha^{*}: \mathfrak{x}_{B^{\prime}} \rightarrow \mathfrak{x}_{B}$ is additive.

Proof. Let $\alpha: B \rightarrow B^{\prime}$ be a morphism in $\mathfrak{B}$ and let $f^{\prime}, g^{\prime}, f^{\prime}+g^{\prime}$ : $X^{\prime} \rightarrow Y^{\prime}$ be morphisms in $\mathfrak{x}_{B^{\prime}}$. Since $\xi_{\alpha}$ is a natural transformation, $\xi_{\alpha}\left(Y^{\prime}\right)\left(\alpha^{*}\left(f^{\prime}+g^{\prime}\right)\right)=\left(f^{\prime}+g^{\prime}\right) \xi_{\alpha}\left(X^{\prime}\right)$. By definition of preadditivity and the naturality of $\xi_{\alpha}, \xi_{\alpha}\left(Y^{\prime}\right)\left(\alpha^{*}\left(f^{\prime}\right)+\alpha^{*}\left(g^{\prime}\right)\right)=\xi_{\alpha}\left(Y^{\prime}\right) \alpha^{*}\left(f^{\prime}\right)+$ $\xi_{\alpha}\left(Y^{\prime}\right) \alpha^{*}\left(g^{\prime}\right)=f^{\prime} \circ \xi_{\alpha}\left(X^{\prime}\right)+g^{\prime} \circ \xi_{\alpha}\left(X^{\prime}\right)=\left(f^{\prime}+g^{\prime}\right) \circ \xi_{\alpha}\left(X^{\prime}\right)$. Hence $\psi=$ $\xi_{\alpha}\left(Y^{\prime}\right)\left(\alpha^{*}\left(f^{\prime}+g^{\prime}\right)\right)=\xi_{\alpha}\left(Y^{\prime}\right)\left(\alpha^{*}\left(f^{\prime}\right)+\alpha^{*}\left(g^{\prime}\right)\right)$. By the uniqueness of a component of $\psi$, we have $\alpha^{*}\left(f^{\prime}+g^{\prime}\right)=\alpha^{*}\left(f^{\prime}\right)+\alpha^{*}\left(g^{\prime}\right)$. Therefore $\alpha^{*}$ is additive.

Definition 2.7. A preadditive fibred category $(\mathfrak{x}, \mathfrak{B}, P$ ) is said to be additive (abelian) iff each fibre is additive (abelian such that for each $\alpha: B \rightarrow B^{\prime}, \alpha^{*}: \mathfrak{x}_{B^{\prime}} \rightarrow *^{*}{ }_{B}$ preserves the analysis of morphisms.)

Definition 2.8. A fibred category $(\mathfrak{x}, \mathfrak{B}, P)$ is said to be multiplicative iff $\mathfrak{X}, \mathfrak{B}$ are multiplicative categories with functors $\otimes: \mathfrak{x} \times \mathfrak{X}$ $\rightarrow \mathfrak{X}, \widetilde{\otimes}: \mathfrak{B} \times \mathfrak{B} \rightarrow \mathfrak{B}$ and with ground objects $K, \widetilde{K}$ respectively, satisfying the following conditions:

1) The diagram

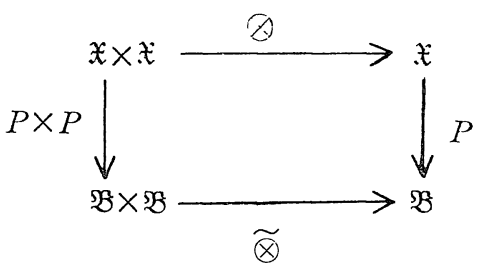

is commutative, 
2) For $\alpha: A \rightarrow A^{\prime}, \beta: B \rightarrow B^{\prime}$ in $\mathfrak{B}$ and for objects $X^{\prime}$ in $\mathfrak{x}_{A^{\prime}}, Y^{\prime}$ in $\mathfrak{x}_{B^{\prime}},\left(\xi_{\alpha}\left(X^{\prime}\right) \otimes \xi_{\alpha}\left(Y^{\prime}\right)\right)^{\prime}: a^{*}\left(X^{\prime}\right) \otimes \beta^{*}\left(Y^{\prime}\right) \rightarrow(\alpha \otimes \beta)^{*}\left(X^{\prime} \otimes Y^{\prime}\right)$ is an isomorphism, where $\left(\xi_{\alpha}\left(X^{\prime}\right) \otimes \xi_{\beta}\left(Y^{\prime}\right)\right)^{\prime}$ is the unique component in $\mathfrak{X}_{A \tilde{\otimes} B}$ of $\xi_{\alpha}\left(X^{\prime}\right) \otimes \xi_{\beta}\left(Y^{\prime}\right)$.

Theorem 2.9. Given a fibred category $(\mathfrak{X}, \mathfrak{S}, P)$ and a functor $\theta: \overline{\mathfrak{B}} \rightarrow \mathfrak{B}$. Then the induced fibred category $\theta^{-1}(\mathfrak{X}, \mathfrak{B}, P)$ inherits the properties of $(\mathfrak{X}, \mathfrak{B}, P)$ and $\theta$; pointed, preadditive, additive, abelian, and multiplicative.

Proof. Let $\bar{\alpha}: \bar{B} \rightarrow \bar{B}^{\prime}$ be a morphism in $\overline{\mathfrak{B}}$, then $\operatorname{hom}_{\bar{\alpha}}((X, \bar{B})$, $\left.\left(X^{\prime}, \bar{B}^{\prime}\right)\right)=\operatorname{hom}_{\theta(\bar{\alpha})}\left(X, X^{\prime}\right) \times \bar{\alpha}$ for $(X, \bar{B}),\left(X^{\prime}, \bar{B}^{\prime}\right)$ in $\theta^{-1}(\mathfrak{X})$. Hence th induce d fibred category is pointed, preadditive, additive, or abelian according as $(\mathfrak{X}, \mathfrak{B}, P)$ is pointed, preadditive, additive or abelian.

Assume that $\theta: \overline{\mathfrak{B}} \rightarrow \mathfrak{B}$ preserves multiplication. Defining $(X, \bar{B})$ $\bar{\otimes}\left(X^{\prime}, \overline{B^{\prime}}\right)=\left(X \otimes X^{\prime}, \bar{B} \bar{\otimes} \bar{B}^{\prime}\right)$ and $(f, \bar{\alpha}) \bar{\otimes}\left(f^{\prime}, \bar{\alpha}^{\prime}\right)=\left(f \otimes f^{\prime}, \bar{\alpha} \bar{\otimes} \bar{\alpha}^{\prime}\right)$ we have a functor $\bar{\otimes}: \theta^{-1}(\mathfrak{X}) \times \theta^{-1}(\mathfrak{X}) \rightarrow \theta^{-1}(\mathfrak{X})$, such that $\bar{P} \circ \bar{\otimes}=\bar{\otimes} \circ\left(\bar{P} \times \overline{Y^{\prime}}\right)$. Let $\bar{K}$ be a ground object in $\overline{\mathscr{K}}$, then $(K, \bar{K})$ is a ground object in $\theta^{-1}(\mathfrak{X})$. For $\bar{\alpha}: \bar{A} \rightarrow \overline{A^{\prime}}, \bar{\beta}: \bar{B} \rightarrow \bar{B}^{\prime}$ in $\overline{\mathfrak{B}}$ and objects $\left(X^{\prime}, \overline{A^{\prime}}\right),\left(Y^{\prime}, \bar{B}^{\prime}\right)$ in $\theta^{-1}(\mathfrak{X})$,

$$
\begin{aligned}
\xi_{\bar{\alpha}}\left(X^{\prime}, \overline{A^{\prime}}\right) \bar{\otimes} \xi_{\bar{\beta}}\left(Y^{\prime}, \bar{B}^{\prime}\right)=\left(\xi_{\theta(\bar{\alpha})}\left(X^{\prime}\right), \bar{\alpha}\right) \bar{\otimes}\left(\xi_{\theta(\bar{\beta})}\left(Y^{\prime}\right), \bar{\beta}\right) \\
=\left(\xi_{\theta(\bar{\alpha})}\left(X^{\prime}\right) \otimes \xi_{(\bar{\beta})}\left(Y^{\prime}\right), \bar{\alpha} \bar{\otimes} \bar{\beta}\right) \\
=\left(\xi_{\theta(\bar{\alpha} \times \bar{\beta})}\left(X^{\prime} \otimes Y^{\prime}\right), \bar{\alpha} \bar{\otimes} \bar{\beta}\right) \circ\left(\left(\xi_{\theta(\bar{\alpha})}\left(X^{\prime}\right) \otimes \xi_{\theta(\bar{\beta})}\left(Y^{\prime}\right)\right)^{\prime},\right.
\end{aligned}
$$

$\left.1_{\overline{A \otimes} \bar{B}}\right)$, so that $\left(\xi_{\bar{\alpha}}\left(X^{\prime}, \overline{A^{\prime}}\right) \bar{\otimes} \xi_{\bar{\beta}}\left(Y^{\prime}, \bar{B}^{\prime}\right)\right)^{\prime}=\left(\left(\xi_{\theta(\bar{\alpha})}\left(X^{\prime}\right) \otimes \xi_{\theta(\bar{\beta})}\left(Y^{\prime}\right)\right)^{\prime}, 1_{\overline{A \otimes} \bar{B}}\right)$ is an isomorphism. Hence $\theta^{-1}(\mathfrak{X}, \mathfrak{B}, P)$ is multiplicative if $(\mathfrak{X}, \mathfrak{B}, P)$ and $\theta$ are multiplicative. The proof is completed.

Corollary 2.10. The fibred product of fibred categories $(\mathfrak{X}, \mathfrak{B}, P$ ), $(\mathfrak{Y}, \mathfrak{B}, Q)$ is pointed, preadditive, additive, abelian, or multiplicative, according as the fibred categories are pointed, preadditive, additive, abelian, or multiplicative.

Proof. The corollary follows immediately from the fact that the fibred product is the induced fibred category $\Delta^{-1}(\mathfrak{X} \times \mathfrak{V}, \mathfrak{B} \times \mathfrak{B}, P \times Q)$ by the diagonal functor $\Delta: \mathfrak{B} \rightarrow \mathfrak{B} \times \mathfrak{B}$ and that the product fibred category $(\mathfrak{X} \times \mathfrak{Y}, \mathfrak{B} \times \mathfrak{B}, P \times Q)$ inherits the above properties of the compo- 
nent fibred categories.

Proposition 2.11. If $(F, \theta):(\mathfrak{X}, \mathfrak{B}, P) \rightarrow(\mathfrak{M}, \mathfrak{A}, S)$ and $(G, \theta)$ :

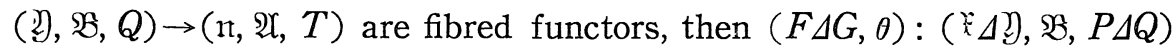
$\rightarrow(\mathfrak{M} \Delta \mathfrak{n}, \mathfrak{A}, S \Delta T)$ defined by $(F \Delta G)(X, Y, B)=(F(X), G(Y), \theta(B))$ and $(F \Delta G)(f, g, \alpha)=(F(f), G(g), \theta(\alpha))$, is a fibred functor.

Proof. Evident.

\section{Category of Fibred Functors}

Definition 3.1. A fibred category $(\mathfrak{V}), \mathfrak{A}, Q)$ is said to have kernels if each fibre $\mathfrak{Y}_{A}$ has kernels such that the functor $\delta^{*}: \mathfrak{Y}_{A^{\prime}} \rightarrow \mathfrak{Y}_{A}$ for each morphism $\delta: A \rightarrow A^{\prime}$ in $\mathfrak{A}$, is kernel preserving.

Definition 3.2. Let $(S, \theta),(T, \theta):(¥, \mathfrak{B}, P) \rightarrow(\mathfrak{Y}, \mathfrak{A}, Q)$ be fibred functors. By a fibred natural transformation $\lambda:(S, \theta) \rightarrow(T, \theta)$ we mean a function which assigns to each object $X$ in $X$ a morphism $\lambda(X)$ : $S(X) \rightarrow T(X)$ in the fibre $\mathfrak{Y}_{\theta P(X)}$ such that $\lambda\left(X^{\prime}\right) \circ S(f)=T(f) \circ \lambda(X)$ for each morphism $f: X \rightarrow X^{\prime}$ in $¥$.

Proposition 3.3. Let $(\dddot{x}, \mathfrak{B}, P$ ) be a fibred category and let (包, $\mathfrak{A}, Q)$ be an additive fibred category with kernels. Consider the category $\left.\mathscr{F}_{\theta}=(\mathfrak{V}), \mathfrak{A}, Q\right)(\mathfrak{X}, \mathfrak{B}, P)$ of fibred functors $(J, \theta)$ from $(\mathfrak{Y}, \mathfrak{B}, P)$ into $(\mathfrak{Y}, \mathfrak{A}, Q$ ) with $\theta: \mathfrak{B} \rightarrow \mathfrak{A}$ fixed and of fibred natural transformations as morphisms. Then $\mathscr{F}_{\theta}$ is pointed, has kernels, has biproducts, and for morphisms $\lambda, \mu$ in $\mathscr{F}_{\theta}, \lambda+\mu$ is defined such that compcsition is distributive.

Proof. For each object $X$ and a morphism $f: X \rightarrow X^{\prime}$ with $P(f)$ $=\alpha$ in $\mathfrak{x}$, a fibred functor $(N, \theta):(\ddot{r}, \mathfrak{B}, P) \rightarrow(\mathfrak{V}, \mathfrak{U}, Q)$ is defined by $N(X)=N_{\theta P(X)}$, a null object in $\mathfrak{V}_{\theta P(X)}$, and by $N(f)=\xi_{\theta(\alpha)}\left(N_{\theta P\left(X^{\prime}\right)}\right)$. Notice that this can be done, because $\theta(\alpha)^{*}$ is a pcinted functor. It is immediate to see that the functor $(N, \theta)$ is, in fact, a null object in the category $\mathscr{F}_{\theta}$. For a morphism $\lambda: S \rightarrow T$ in $\mathscr{F}_{\theta}$, the kernel $k: K$ $\rightarrow S$ of $\lambda$ is defined as follows. For each object $X$ in $X$ let $k(X)$ : $K(X) \rightarrow S(X)$ be the kernel of $\lambda(X)$ in $\mathfrak{Y}_{\theta P(X)}$. For each morphism 
$f: X \rightarrow X^{\prime}$ in $\mathfrak{X}$ with $P(f)=\alpha$ consider the diagram

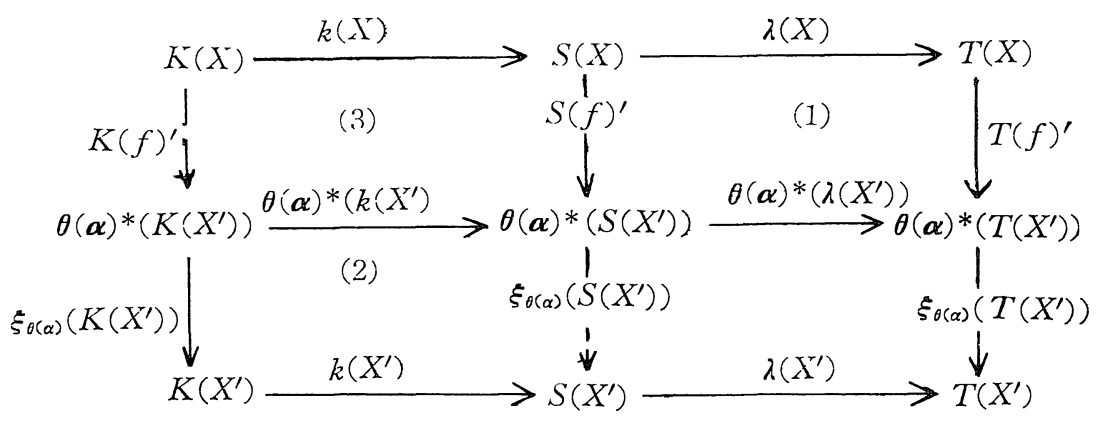

where $S(f)=\xi_{\theta(\alpha)}\left(S\left(X^{\prime}\right)\right) \circ S(f)^{\prime}$ and $T(f)=\xi_{\theta(\alpha)}\left(T\left(X^{\prime}\right)\right) \circ T(f)^{\prime}$. Since $\theta(\alpha)^{*}$ preserves kernels, $\theta(\alpha) *\left(k\left(X^{\prime}\right)\right)$ is the kernel of $\theta(\alpha)^{*}\left(\lambda\left(X^{\prime}\right)\right)$. As the diagram (1) is commutative, there exists a unique morphism $K(f)^{\prime}$ in $\mathfrak{Y}_{\theta F(X)}$ such that the diagram (3) is commutative. Define $K(f)=$ $\xi_{\theta(\alpha)}\left(K\left(X^{\prime}\right)\right) \circ K(f)^{\prime}$, then $k\left(X^{\prime}\right) \circ K(f)=S(f) \circ k(X)$ because of the commutativity of the diagram (2). It is straight forward to verify that $k: K \rightarrow S$ is the kernel of $\lambda$ in the category $\mathscr{F}_{\theta}$. For objects $S, T$ in $\mathscr{F}_{\theta}$ and for each object $X$ in $X$, there exists the biproduct $S(X)+$ $T(X)$ in the additive category $\mathfrak{Y}_{\theta P(X)}$, together with morphisms $p(S(X))$ : $S(X)+T(X) \rightarrow S(X), \quad p(T(X)): S(X)+T(X) \rightarrow T(X), \quad,(S(X)):$ $S(X) \rightarrow S(X)+T(X)$, and $\iota(T(X)): T(X) \rightarrow S(X)+T(X)$ satisfying the usual conditions. Defining $(S+T)(X)=S(X)+T(X)$ for each object $X$ in $\mathfrak{X}$ and $(S+T)(f)={ }_{\iota}\left(T\left(X^{\prime}\right)\right) \circ T(f) \circ p(T(X))+$ $\iota\left(S\left(X^{\prime}\right)\right) \circ S(f) \circ p(S(X))$ for each morphism $f: X \rightarrow X^{\prime}$ in $\mathfrak{X}$, it can be shown that $S+T$ is the biproduct with morphisms $\iota_{S}: S \rightarrow S+T, \iota_{T}:$ $T \rightarrow S+T, p_{s}: S+T \rightarrow S$ and $p_{T}: S+T \rightarrow T$ defined by $\iota_{S}(X)=\imath(S(X))$, $\iota_{T}(X)=\iota(T(X)), \quad p_{S}(X)=p(S(T))$, and $p_{T}(X)=p(T(X))$. It is obvious that addition of morphisms $\lambda, \mu: S \rightarrow T$ is defined by $(\lambda+\mu)(X)$ $=\lambda(X)+\mu(X)$ for each $X$ in $\mathfrak{X}$ so that composition is distributive. Hence the proof is completed.

Proposition 3.4. Let $(G, \varepsilon, \Delta)$ be a cotriple on a fibred category $(\mathfrak{X}, \mathfrak{B}, P$ ) and let $(\mathfrak{Y}, \mathfrak{A}, Q$ ) be a preadditive fibred category with kernels. Then there exists a cotriple $(\widetilde{G}, \widetilde{\varepsilon}, \widetilde{\Delta})$ on the functor category 
$\mathscr{L}_{\theta}=(\mathfrak{Y}, \mathfrak{A}, Q)(\mathfrak{x}, \mathfrak{B}, P)$ by setting $\widetilde{G}(S)=S G, \widetilde{G}(\lambda)=\lambda G, \widetilde{\varepsilon}(S)=S \varepsilon$, and $\widetilde{\Delta}(S)=S \Delta$ for each object $S$ in $\mathscr{F}_{\theta}$ and each morphism $\lambda$ in $\mathscr{F}_{\theta}$. Moreover the functor $\widetilde{G}: \mathscr{F}_{\theta} \rightarrow \mathscr{F}_{\theta}$ is additive and kernel preserving.

Proof. By definition of a cotriple $(G, \varepsilon, \Delta)$ on a fibred category, $G$ is a fibred functor and $\varepsilon, \Delta$ are fibred natural transformations. Hence it is immediate to see that $(\widetilde{G}, \widetilde{\varepsilon}, \widetilde{\Delta})$ is a cotriple on $\mathscr{F}_{\theta}$. For morphisms $\lambda, \mu$ in $\mathscr{F}_{\theta}$ and for each object $X, \widetilde{G}(\lambda+\mu)(X)=(\lambda+\mu)(G(X))=$ $\lambda(G(X))+\mu(G(X))=\widetilde{G}(\lambda)(X)+\widetilde{G}(\mu)(X)=(\widetilde{G}(\lambda)+\widetilde{G}(\mu))(X)$. Hence $\widetilde{G}$ is additive. Let $k: K \rightarrow S$ be the kernel of $\lambda: S \rightarrow T$. Since $k(G(X))$ is the kernel of $\lambda(G(X))$ for each object $X$ in $x, \widetilde{G}(k)=k G$ is the kernel of $\widetilde{G}(\lambda)$ by definition. Hence $\widetilde{G}$ is kernel preserving.

In the rest of the present section we shall use some of the results of our previous paper [6]. Since the category $\left.\mathscr{F}_{\theta}=(\mathfrak{V}), \mathfrak{A}, Q\right)(\mathfrak{X}, \mathfrak{B}, P)$ is pointed in case $(\mathfrak{Y}, \mathfrak{Y}, Q)$ is pointed fibred category, and since a cotriple $(\widetilde{G}, \tilde{\varepsilon}, \widetilde{\Delta})$ can be defined on $\mathscr{F}_{\theta}$ when a cotriple $(G, \varepsilon, \Delta)$ is given on $(\mathfrak{X}, \mathfrak{B}, P)$, a projective class $\widetilde{\mathcal{E}}$ of sequences in $\mathscr{F}_{\theta}$ can be obtained by $\widetilde{\mathscr{Q}}^{\prime} \Rightarrow \widetilde{\mathcal{E}}$, where $\widetilde{\mathscr{L}}^{\prime}$ is the class of all objects $\widetilde{G}(S)$ for $S$ in $\mathcal{F}_{\theta}$.

Proposition 3.5. Let $\mathcal{E}_{0}\left(\mathscr{F}_{\theta}\right)$ be the class of all split exact sequences in $\mathscr{L}_{\theta}$. Then we have $\widetilde{\mathcal{E}}=\widetilde{G}^{-1}\left(\mathcal{E}_{0}\left(\mathscr{F}_{\theta}\right)\right)$.

Proof. This proposition is proved in a similar manner as Proposition 1.2 in [6].

Let $\widetilde{G}$ be the standard semi-simplicial complex of the cotriple $(\widetilde{G}$, $\bar{\varepsilon}, \widetilde{\Delta})$ on $\mathscr{F}_{\theta}$. Then we have

Theorem 3.6. For each object $S$ in $\mathscr{F}_{\theta}$ the augmented chain complex $\widetilde{G}(S) \stackrel{\widetilde{\varepsilon}(S)}{\longrightarrow} S$ is a $\widetilde{G}^{-1}\left(\mathcal{E}_{0}\left(\mathscr{F}_{\theta}\right)\right)$-projective resolution of $S$ in $\mathscr{F}_{\theta}$.

Proof. One can prove this theorem by referring to Theorem 2.2 and Proposition 5.1 in [3] and by referring to Theorem 3.1 and Corollary 3.2 in $[6]$.

\section{Eilenberg-Zilber Theorem}

Definition 4.1. A fibred category $(\mathfrak{X}, \mathfrak{B}, P)$ is called a tensored 
fibred category iff it is additive and multiplicative such that the multiplication functors $\otimes: \mathfrak{X} \times \mathfrak{X} \rightarrow \mathfrak{X}$ and $\widetilde{\otimes}: \mathfrak{B} \times \mathfrak{B} \rightarrow \mathfrak{B}$ are biadditive.

It is immediate to see that a tensored fibred category $(\mathfrak{X}, \mathfrak{B}, P)$ satisfies the properties; 1) $\left(X_{1}+X_{2}\right) \otimes X^{\prime}=X_{1} \otimes X^{\prime}+X_{2} \otimes X^{\prime}, X \otimes\left(X_{1}^{\prime}\right.$ $\left.+X_{2}^{\prime}\right)=X \otimes X_{1}^{\prime}+X \otimes X_{2}^{\prime}$, whenever they are well defined, 2) $N \otimes X^{\prime}$ and $X \otimes N^{\prime}$ are null objects if $N, N^{\prime}$ are null objects.

Let $\mathbb{B}=(\mathfrak{R}, \mathfrak{B}, P)(\mathfrak{X}, \mathfrak{B}, P)$ be the category of fibred functors of the form $\left(S, 1_{\mathfrak{B}}\right)$ and let $\mathscr{F}_{\tilde{N}}=(\mathfrak{X}, \mathfrak{B}, P)(\mathfrak{X} \times \mathfrak{X}, \mathfrak{B} \times \mathfrak{B}, P \times P)$. Then $\mathfrak{B}$ and $\mathscr{F}_{\tilde{\Phi}}$ are pointed, have kernels and biproducts, if the tensored fibred category $(\mathfrak{X}, \mathfrak{B}, P)$ has kernels.

For objects $S, T$ in $(\mathcal{S}$, an object $(S, T)$ in $(\mathfrak{X} \times \mathfrak{X}, \mathfrak{B} \times \mathfrak{B}, P \times$ $P)(\mathfrak{X} \times \mathfrak{X}, \mathfrak{B} \times \mathfrak{B}, P \times P)$ is defined by $(S, T)\left(X, X^{\prime}\right)=\left(S(X), T\left(X^{\prime}\right)\right)$ and $(S, T)(f, g)=(S(f), T(g))$ for objects $\left(X, X^{\prime}\right)$ and morphisms $(f$, $g)$. Then an object in $\mathscr{F} \tilde{\otimes}$, denoted by $S \otimes T$, is obtained by $(\otimes \circ(T$, $S$ ), $\widetilde{\otimes})$. In a similar manner, for morphisms $\lambda: T \rightarrow T^{\prime}, \mu: S \rightarrow S^{\prime}$ in (S), a morphism in $\mathcal{F}_{\tilde{\otimes}}$ denoted by $\lambda \otimes \mu$, can be defined by $\otimes \circ(\lambda, \mu)$ : $T \otimes S \rightarrow T^{\prime} \otimes S^{\prime}$. Then it is easy to see that in the category $\mathscr{F}_{\tilde{}}$ we have $\left(T_{1}+T_{2}\right) \otimes S=T_{1} \otimes S+T_{2} \otimes S, \quad T \otimes\left(S_{1}+S_{2}\right)=T \otimes S_{1}+T \otimes S_{2}, \quad(\lambda$ $+\mu) \otimes \nu=\lambda \otimes \nu+\mu \otimes \nu$, and $\lambda \otimes(\mu+\nu)=\lambda \otimes \mu+\lambda \otimes \nu$ for objects $T, S, T_{1}$, $T_{2}, S_{1}, S_{2}$ and morphisms $\lambda, \mu, \nu$ in (S). Let us see this quickly. For each object $\left(X, X^{\prime}\right)$ in $\mathfrak{x} \times \mathfrak{X},\left(\left(T_{1}+T_{2}\right) \otimes S\right)\left(X, X^{\prime}\right)=\otimes \circ\left(T_{1}+T_{2}\right)$, $\left.S))\left(X, X^{\prime}\right)=\otimes\left(T_{1}(X)+T_{2}(X), S\left(X^{\prime}\right)\right)=T_{1}(X)+T_{2}(X)\right) \otimes S\left(X^{\prime}\right)$ $=T_{1}(X) \otimes S\left(X^{\prime}\right)+T_{2}(X) \otimes S\left(X^{\prime}\right)=\left(T_{1} \otimes S+T_{2} \otimes S\right)\left(X, X^{\prime}\right) . \quad((\lambda+$ $\mu) \otimes \nu)\left(X, X^{\prime}\right)=\otimes\left((\lambda+\mu)(X), \nu\left(X^{\prime}\right)\right)=\otimes\left(\lambda(X)+\mu(X), \nu\left(X^{\prime}\right)\right)=\otimes$ $\left(\lambda(X), \nu\left(X^{\prime}\right)\right)+\otimes\left(\mu(X), \nu\left(X^{\prime}\right)\right)=(\lambda \otimes \nu+\mu \otimes \nu)\left(X, X^{\prime}\right)$.

Given a cotriple $(F, \eta, \rho)$ on the tensored category $(\mathfrak{X}, \mathfrak{B}, P)$ with kernels. From Proposition 3.4 and from the observation made above, the cotriple induces a cotriple $(\widetilde{F}, \tilde{\eta}, \tilde{\rho})$ on $(5)$, and a cotriple $((F, F)$, $(\eta, \eta),(\rho, \rho))$ on $(\mathfrak{X} \times \mathfrak{X}, \mathfrak{B} \times \mathfrak{B}, P \times P)$ so that a cotriple $((\widetilde{F, F}),(\tilde{\eta}, \eta)$, $(\tilde{\rho, \rho}))$ on $\mathscr{F} \otimes$. $(F, F),(\eta, \eta),(\rho, \rho)$ will be denoted by $G, \varepsilon$, and $\Delta$ respectively. From Proposition $3.5, \widetilde{F}^{-1}\left(\mathcal{E}_{0}(\mathcal{B})\right)$ and $\widetilde{G}^{-1}\left(\mathcal{E}_{0}(\mathscr{F} \tilde{\otimes})\right)$ are projective classes in $(\mathscr{S})$ and $\mathscr{F} \otimes$ respectively, where $\mathcal{E}_{0}($ ) denotes the appropriate class of all split exact sequences. 
In view of Theorem 3.6 the augmented chain complex $\mathbb{F} \stackrel{n}{\longrightarrow} I$;

$$
\cdots \rightarrow F^{n+1} \stackrel{\partial_{u}}{\longrightarrow} F^{n} \rightarrow \cdots \stackrel{\partial_{1}}{\longrightarrow} F \stackrel{n}{\longrightarrow} I
$$

is a $\widetilde{F}^{-1}\left(\mathcal{E}_{0}(\&)\right)$-projective resolution of the identity functor $\left(I, 1_{\mathfrak{B}}\right)$ in (8), where $\partial_{n}=\sum_{i=0}^{n}(-1)^{i} \partial_{n}^{i}$ with $\partial_{n}^{i}=F^{n-1} \eta F^{i}$ for each $i$. Hence the complex $\widetilde{F}(\mathbb{F} \stackrel{n}{\longrightarrow} I)=\widetilde{F}(\boldsymbol{F}) \stackrel{\eta_{F}}{\longrightarrow} F$ has a contracting homotopy $S_{n}: F^{n+2}$ $\rightarrow F^{n+3}$ for each $n \geq-1$ in the category \&S. Let us consider two augmented chain comlexes $\boldsymbol{F} \times \mathbb{F}, \mathbb{F} \otimes \boldsymbol{F}$ over $I \otimes I$ in the category $\mathscr{F}_{\tilde{E}}$ as follows. The first one $F \times \mathbb{F} \stackrel{\eta \otimes \eta}{\longrightarrow} I \otimes I$ is defined by

$$
\cdots \rightarrow F^{n+1} \otimes F^{n+1} \stackrel{D^{n}}{\longrightarrow} F^{n} \otimes F^{n} \rightarrow \cdots \stackrel{D_{1}}{\longrightarrow} F \otimes F \rightarrow I \otimes I,
$$

where $D_{n}=\sum(-1)^{i} \partial_{n}^{i} \otimes \partial_{n}^{i}$. The other one $F \otimes F \stackrel{\eta \otimes n}{\longrightarrow} I \otimes I$ is defined by $(\boldsymbol{F} \otimes \boldsymbol{F})_{n}=\sum_{\substack{p+q=n \\ p, q \geq 0}} F^{p+1} \otimes F^{q+1}$ such that the $n$-th boundary $d_{n}:(\boldsymbol{F} \otimes \boldsymbol{F})_{n}$ $\rightarrow(\boldsymbol{F} \otimes \boldsymbol{F})_{n-1}$ is given by $\sum_{p, q}\left(\iota_{p, q+1}\left(\partial_{p} \otimes 1\right)+(-1)^{p} \iota_{p+1, q}\left(1 \otimes \partial_{q}\right)\right) \pi_{p+1, q+1}$ where $\pi_{p+1, q+1}:(\boldsymbol{F} \otimes \boldsymbol{F})_{n} \rightarrow F^{p+1} \otimes F^{q+1}$ is a projection morphism and $\iota_{p+1, q}$ : $F^{p+1} \otimes F^{q} \rightarrow(\boldsymbol{F} \otimes \boldsymbol{F})_{n-1}$ is an injection morphism. As is usually the case, both $\boldsymbol{F} \times \boldsymbol{F}$ and $\boldsymbol{F} \otimes \boldsymbol{F}$ form complexes in $\mathscr{F}_{\tilde{E}}$.

Theorem 4.2. The augmented complexes $\boldsymbol{F} \times \mathbb{F} \stackrel{\eta \otimes n}{\longrightarrow} I \otimes I$ and $\boldsymbol{F} \otimes$ $\boldsymbol{F} \stackrel{n \otimes n}{\longrightarrow} I \otimes I$ are $\widetilde{G}^{-1}\left(\mathcal{E}_{0}\left(\mathscr{F}_{\tilde{t}}\right)\right)$-projective resolutions of $I \otimes I=\otimes$ in $\mathscr{F}_{\tilde{\imath}}$.

Proof. In view of Theorem 3.6, $\widetilde{G}(\otimes) \stackrel{\tilde{\varepsilon}(\otimes)}{\longrightarrow} \otimes$ is a $\widetilde{G}^{-1}\left(\mathcal{E}_{0}\left(\mathscr{F}_{\tilde{k}}\right)\right)$. projective resolution of $\otimes$. Since $\widetilde{G}(\otimes)_{n}=\widetilde{G}^{n+1}(\otimes)=\otimes G^{n+1}=\otimes\left(F^{n+1}\right.$, $\left.F^{n+1}\right)=F^{n+1} \otimes F^{n+1}$ and since the $n$-th boundary operator $\alpha_{n}(\otimes): \widetilde{G}^{n+1}(\otimes)$ $\rightarrow \widetilde{G}^{n}(\otimes)$ is $\sum(-1)^{i} \alpha_{n}^{i}(\otimes)$ such that $\alpha_{n}^{i}(\otimes)=\otimes \alpha_{n}^{i}=\otimes\left(G^{n-i} \epsilon G^{i}\right)=$ $\otimes\left(F^{n-i} \eta F^{i}, F^{n-i} \eta F^{i}\right)=\otimes\left(\partial_{n}^{i}, \hat{\partial}_{n}^{i}\right)=\partial_{n}^{i} \otimes \partial_{n}^{i}, \boldsymbol{F} \times \boldsymbol{F} \stackrel{n \otimes n}{\longrightarrow} I \otimes I$ is a $\widetilde{G}^{-1}\left(\mathcal{E}_{0}\left(\mathcal{F}_{\tilde{\Psi}}\right)\right)$ -projective resolution of $\otimes$ in $\mathscr{F} \approx$.

As $\widetilde{G}: \mathscr{F}_{\widetilde{\mho}} \rightarrow \mathscr{F}_{\tilde{\mho}}$ is additive, $(\boldsymbol{F} \otimes \boldsymbol{F})_{n}=\sum_{\substack{p+q=n \\ p, q \geq 0}} F^{p+1} \otimes F^{q+1}=\sum \otimes\left(F^{p}\right.$, $\left.F^{q}\right)(F, F)=\sum\left(F^{p} \otimes F^{q}\right) G=\sum \widetilde{G}\left(F^{p} \otimes F^{a}\right)=\widetilde{G}\left(\sum F^{p} \otimes F^{a}\right)$. Hence $(\boldsymbol{F}$ $\otimes \boldsymbol{F})^{n}$ is $\widetilde{G}^{-1}\left(\mathcal{E}_{0}\left(\mathscr{F}_{\tilde{k}}\right)\right)$-projective for each $n \geq 0$. The proof will be completed if the complex $\widetilde{G}(\boldsymbol{F} \otimes \boldsymbol{F}) \stackrel{\eta \otimes \eta}{\longrightarrow} I \otimes I)=\widetilde{G}(F \otimes F) \stackrel{\eta F \otimes \eta F}{\longrightarrow} F \otimes F$ is $\widetilde{G}^{-1}\left(\mathcal{E}_{0}\left(\mathscr{F}_{\tilde{k}}\right)\right)$-acyclic. Since $\widetilde{G}(\boldsymbol{F} \otimes \boldsymbol{F})=\widetilde{F}(\boldsymbol{F}) \otimes \widetilde{F}(\boldsymbol{F})$ and since $\widetilde{F}(\boldsymbol{F}) \stackrel{\eta F}{\longrightarrow} F$ has a contracting homotopy $\left\{s_{n} \mid n \geq-1\right\}, \widetilde{G}(\boldsymbol{F} \otimes \boldsymbol{F}) \stackrel{\eta F \otimes \eta F}{\longrightarrow} F$ $\otimes F$ also has a contracting homotopy $\left\{S_{n} \mid n \geq-1\right\}$ defined by $\sum l_{p+3, q+2}$ 
$\left(s_{p} \otimes 1\right) \pi_{p+2, q+2} \sum \iota_{p+2, q+3}\left(s_{-1} \eta F \otimes s_{q}\right) \pi_{p+2, q+2}$ for $n \geq 0$ and $S_{-1}=s_{-1} \otimes s_{-1}$, where $S_{n}: \sum_{\substack{p+q=n \\ p, q \geq 0}} F^{p+2} \otimes F^{q+2} \underset{\substack{\gamma+s=n+1 \\ \gamma, s \geq 0}}{\rightarrow} \sum^{\gamma+2} \otimes F^{s+2}$ and $S_{-1}: F \otimes F \rightarrow F^{2} \otimes F^{2}$.

\section{Product}

Let $(\mathfrak{X}, \mathfrak{B}, P)$ be a fibred category on which a cotriple $(G, \varepsilon, \Delta)$ is defined, and let $(\mathfrak{Y}, \mathfrak{A}, Q)$ be a tensored fibred category with kernels where $\widehat{\otimes}: \mathfrak{Y} \times \mathfrak{Y} \rightarrow \mathfrak{Y}$ and $\widetilde{\otimes}: \mathfrak{U} \times \mathfrak{X} \rightarrow \mathfrak{N}$ are the multiplication functors. If $(T, \theta):(\Re, \mathfrak{B}, P) \rightarrow(\mathfrak{Y}, \mathfrak{A}, Q)$ is a covariant (contravariant) functor, then the cotriple induces a cotriple $(\widetilde{G}, \widetilde{\varepsilon}, \widetilde{\Delta})$ on the category $\mathscr{F}_{\theta}=(\mathfrak{V})$, $\mathfrak{A}, Q)(\mathfrak{x}, \mathfrak{B}, P)$ and a cotriple $((\widetilde{G, G}),(\widetilde{\varepsilon, \varepsilon}),(\widetilde{\Delta, \Delta}))$ on the category $\left.\mathscr{F}_{\bar{\Theta}(\theta, \theta)}=(\mathfrak{V}), \mathfrak{A}, P\right)(\mathfrak{X} \times \mathfrak{x}, \mathfrak{\wp} \times \mathfrak{B}, P \times P)$. Let us denote the standard semisimplicial complex associated with $(G, \varepsilon, \Delta)$ by

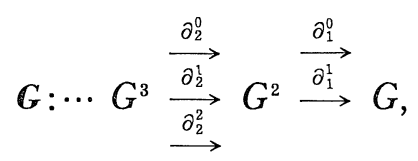

where $\partial_{n}^{i}: G^{n+1} \rightarrow G^{n}$ is $G^{n-1} \varepsilon G^{i}$, and let $T G \stackrel{T \varepsilon}{\longrightarrow} T$ be an augmented chain complex in $\mathscr{F}_{\theta}$ defined by $T G: \cdots \rightarrow T G^{n+1} \stackrel{\partial^{n}}{\longrightarrow} T G^{n} \rightarrow \cdots \stackrel{\partial_{1}}{\longrightarrow} T G \stackrel{T \varepsilon}{\longrightarrow} T$, where $\partial_{n}=\sum_{i=0}^{n}(-1)^{i} T \partial_{n}^{i}$. Then we have

Proposition 5.1. The augmented chain complex $T G \stackrel{T_{n}}{\longrightarrow} T$ is a $\widetilde{G}^{-1}\left(\mathcal{E}_{0}\left(\mathscr{F}_{\theta}\right)\right)$-projective resolution of $T$ in $\mathscr{F}_{\theta}$. The chain complexes

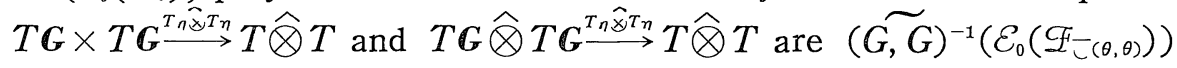
-projective resolutions of $T \otimes T$ in $\mathscr{F}_{E}(\theta, \theta)$. Since the proof is similar to that of Theorem 4.2 , it is omitted.

Given a cotriple $(G, \varepsilon, \Delta)$ on a multiplicative fibred category ( $\ddot{x}$, $\mathfrak{B}, P)$ with the multiplication functors $\otimes: \mathfrak{X} \times \mathfrak{X} \rightarrow \mathfrak{X}, \widetilde{\otimes}: \mathfrak{B} \times \mathfrak{B} \rightarrow \mathfrak{B}$. Then there exist semi-simplicial complexes $\boldsymbol{G} \times \boldsymbol{G}, \boldsymbol{G} \otimes$ defined by

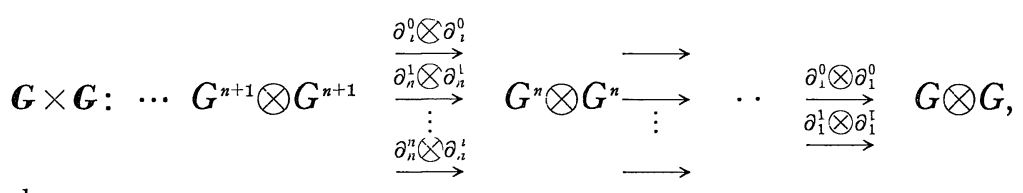

and 


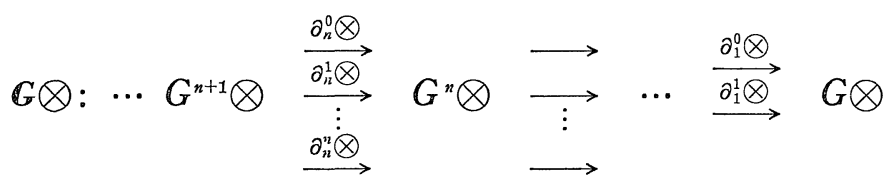

where $\partial_{n}^{i}=G^{n-i} \epsilon G^{i}(i=0, \cdots, n)$. Then

Proposition 5.2. If there exists a natural transformation $\mu: G \otimes$ $G \rightarrow G \otimes$ such that the diagram

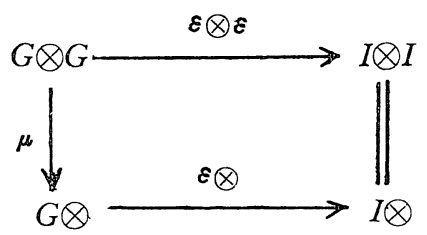

is commutative, then there exists a semi-simplicial map $\bar{\mu}: \mathbb{G} \times \boldsymbol{G} \rightarrow \boldsymbol{G} \otimes$ satisfying the conditions that

for each $i$.

$$
\bar{\mu}_{0}=\mu, \quad\left(\partial_{n}^{i} \otimes\right) \circ \bar{\mu}_{n}=\bar{\mu}_{n-i} \circ\left(\partial_{n}^{i} \otimes \partial_{n}^{i}\right)
$$

Proof. Let us define inductively $\bar{\mu}_{0}=\mu$ and $\bar{\mu}_{n}=G \bar{\mu}_{n-i} \circ \mu\left(G^{n}, G^{n}\right)$ for each $n \geq 0$. Since $\mu$ is a natural transformation, we have a commutative diagram

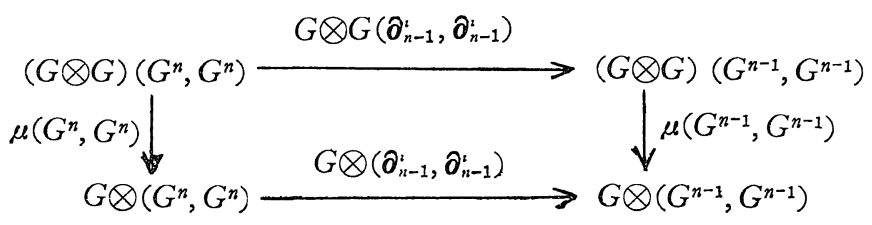

for each $n>i \geq 0$. Assume that $\left(\partial_{n-1}^{i} \otimes\right) \circ \bar{\mu}_{n-1}=\bar{\mu}_{n-2} \circ\left(\partial_{n-1}^{i} \otimes \partial_{n-1}^{i}\right)$. Then $\left(\partial_{n}^{i} \otimes\right) \circ \bar{\mu}_{n}=\left(\partial_{n}^{i} \otimes\right) \circ G \bar{\mu}_{n-1} \circ \mu\left(G^{n}, G^{n}\right)=G\left(\partial_{n-1}^{i} \otimes \circ \bar{\mu}_{n-1}\right) \circ \mu\left(G^{n}, G^{n}\right)=G\left(\bar{\mu}_{n-2} \circ\right.$ $\left.\left(\partial_{n-1}^{i} \otimes \partial_{n-1}^{i}\right)\right) \circ \mu\left(G^{n}, G^{n}\right)=G \bar{\mu}_{n-2} \circ G\left(\partial_{n-1}^{i} \otimes \partial_{n-1}^{i}\right) \circ \mu\left(G^{n}, G^{n}\right)=G \bar{\mu}_{n-2} \circ \mu\left(G^{n-1}\right.$, $\left.G^{n-1}\right) \circ\left(\partial_{n}^{i} \otimes \partial_{n}^{i}\right)=\bar{\mu}_{n-1} \circ\left(\partial_{n}^{i} \otimes \partial_{n}^{i}\right)$. Similarly $\left(\partial_{n}^{n} \otimes\right) \circ \bar{\mu}=\bar{\mu}_{n-1} \circ\left(\partial_{n}^{n} \otimes \partial_{n}^{n}\right)$ can be shown. Hence the proof is completed.

Proposition 5.3. Given a cotriple $(G, \varepsilon, \Delta)$ on a multiplicative fibred category $(\mathfrak{X}, \mathfrak{B}, P)$ such that there exists a natural transformation $\mu: G \otimes G \rightarrow G \otimes$ satisfying $(\varepsilon \otimes) \circ \mu=\varepsilon \otimes \varepsilon$. Let $(\mathfrak{y}, \mathfrak{X}, Q$ ) be a pre- 
additive fibred category and let $(T, \theta):(\mathfrak{X}, \mathfrak{B}, P) \rightarrow(\mathfrak{Y}, \mathfrak{X}, Q)$ be a functor. Then there exists a chain map $T_{\bar{\mu}}: T(G \times G) \rightarrow T G \otimes$ in the category $\mathscr{F}_{\theta^{\sim}}$, where $\widetilde{\otimes}: \mathfrak{B} \times \mathfrak{B} \rightarrow \mathfrak{B}$.

Proof. Consider the diagram

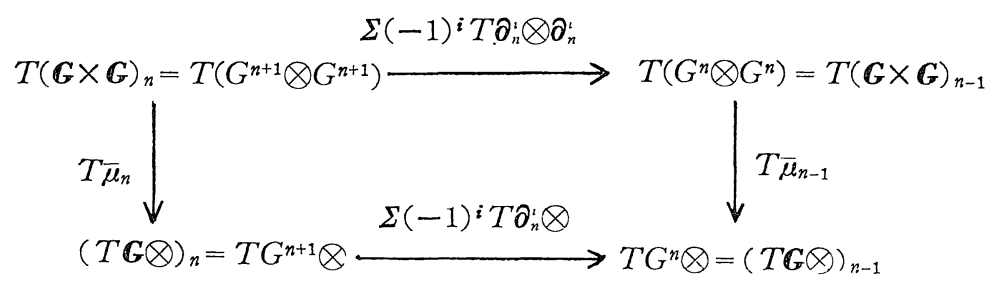

then by Proposition $5.2\left(\sum(-1)^{i} T \partial_{n}^{i}\right) \circ\left(T \bar{\mu}_{n}\right)=\sum(-1)^{i}\left(T \partial_{n}^{i}\right) \circ\left(T \bar{\mu}_{n}\right)=$ $\sum(-1)^{i} T\left(\partial_{n}^{i} \circ \bar{\mu}_{n}\right)=\sum(-1)^{i} T\left(\bar{\mu}_{n-1} \circ\left(\partial_{n}^{i} \otimes \partial_{n}^{i}\right)\right)=T \bar{\mu}_{n-1} \circ\left(\sum(-1)^{i} T \partial_{n}^{i} \otimes \partial_{n}^{i}\right)$. Hence the proof is completed.

Proposition 5.4. Let $(\mathfrak{X}, \mathfrak{B}, P)$ be a multiplicative fibred category and let $(\mathfrak{Y}, \mathfrak{R}, Q)$ be a tensored category with kernels. Given a functor $(T, \theta):(\mathfrak{X}, \mathfrak{B}, P) \rightarrow(\mathfrak{Y}, \mathfrak{X}, Q)$ such that there exists a natural transformation $\nu: \hat{\otimes} \circ(T, T) \rightarrow T \otimes$, where $\otimes: \mathfrak{X} \times \mathfrak{X} \rightarrow \mathfrak{X}, \widehat{\otimes}: \mathfrak{Y} \times \mathfrak{Y} \rightarrow \mathfrak{Y}$ are multiplication functors. If a cotriple $(G, \varepsilon, \Delta)$ is given on $(\mathfrak{X}, \mathfrak{B}, P)$, then there exists a chain map $\bar{\nu}: T \boldsymbol{G} \times T \boldsymbol{G} \rightarrow T(\boldsymbol{G} \times \boldsymbol{G})$ over $\nu$ in the category $\mathscr{F}_{\theta \tilde{\omega}}=\mathscr{F}_{\bar{\otimes}(\theta, \theta)}$, where $\widetilde{\otimes}: \mathfrak{B} \times \mathfrak{B} \rightarrow \mathfrak{B}$ and $\bar{\otimes}: \mathfrak{A} \times \mathfrak{A} \rightarrow \mathfrak{A}$ are multiplication functors. Any two such chain maps over $\nu$ are chain homotopic.

Proof. Since $T \boldsymbol{G} \times T \boldsymbol{G} \stackrel{T \varepsilon \otimes T \varepsilon}{\longrightarrow} T \widehat{\otimes} T$ is a $(\widetilde{G, G})^{-1}\left(\mathcal{E}_{0}\left(\mathscr{F}_{\theta_{\hat{\diamond}}}\right)\right)$-projective resolution of $T \hat{\otimes} T$, and since $T(\boldsymbol{G} \times \boldsymbol{G}) \stackrel{T(\varepsilon ? \xi)}{\longrightarrow} T \otimes$ is a $(\widetilde{G, G})^{-1}$ $\left(\mathcal{E}_{0}\left(\mathcal{F}_{\theta^{2}}\right)\right)$-projective resolution of $T \otimes$, a morphism $\nu: T \hat{\otimes} T \rightarrow T \otimes$ in the category $\mathscr{F}_{\theta \tilde{\sim}}$ can be extended to a chain map $\bar{\nu}: T \boldsymbol{G} \times T \boldsymbol{G} \rightarrow T(\boldsymbol{G}$ $\times \boldsymbol{G})$. It is evident that two such maps are chain homotopic by the usual argument in homological algebra. Hence the proof is completed.

Gathering together Propositions 5.1-5.4, we have

Theorem 5.5. Let $(G, \varepsilon, \Delta)$ be a cotriple defined on a multiplicative fibred category $(\mathfrak{X}, \mathfrak{B}, P)$ such that there exists a natural transformation $\mu: G \otimes G \rightarrow G \otimes$ satisfying $(\varepsilon \otimes) \circ \mu=\varepsilon \otimes \varepsilon$, where $\otimes: \mathfrak{X} \times \mathfrak{X} \rightarrow$ $\mathfrak{X}$. Let $(T, \theta):(\mathfrak{X}, \mathfrak{B}, P) \rightarrow(\mathfrak{Y}, \mathfrak{X}, Q)$ be a covariant (contravariant) 
functor of the fibred category $(\mathfrak{X}, \mathfrak{B}, P)$ into a multiplicative abelian fibred category $(\mathfrak{Y}, \mathfrak{N}, Q)$ such that a natural transformation $\nu: \widehat{\otimes} \circ(T$, $T) \rightarrow T \otimes$ is given, where $\widehat{\otimes}: \mathfrak{Y} \times \mathfrak{Y} \rightarrow \mathfrak{Y}$. Then there exists an external product in the cotriple homology (cohomology) $H_{*}(T \boldsymbol{G})$ : for integers $p, q$ there exists a morphism $\varphi_{p, q}$ in $\mathscr{F}_{\theta^{-}}=(\mathfrak{V}, \mathfrak{A}, Q)(\mathfrak{X} \times \mathfrak{W}, \mathfrak{B} \times \mathfrak{B}, P \times P)$ with $\widetilde{\otimes}: \mathfrak{B} \times \mathfrak{B} \rightarrow \mathfrak{B}$ such that $\varphi_{p, q}: H_{p}(T \mathbb{G}) \hat{\otimes} H_{q}(T \boldsymbol{G}) \rightarrow H_{p+q}(T \mathbb{G} \otimes)$.

Proof. From Proposition 5.1 there is an isomorphism $\alpha_{p+q}: H_{p+q}(T \boldsymbol{G}$ $\widehat{\otimes} T G) \rightarrow H_{t+q}(T G \times T G)$. From Proposition 5.4, $\bar{\nu}$ induces a morphism $\bar{\nu}_{p+q}: H_{p+q}(T \boldsymbol{G} \times T \mathbb{G}) \rightarrow H_{p+q}(T(\mathbb{G} \times \boldsymbol{G}))$, while $T_{\bar{\mu}}$ induces $T_{\bar{\mu}_{p+q}: H_{p+q}}$ $(T(\boldsymbol{G} \times \mathbb{G})) \rightarrow H_{p+q}(T G \otimes)$ in view of Proposition 5.3. Since there always exists a morphism $\iota_{p, q}: H_{p}(T G) \hat{\otimes} H_{q}(T G) \rightarrow H_{p+q}(T \mathbb{G} \hat{\otimes} T G)$, the composite $\varphi_{p, q}=T_{\bar{\mu}_{p+q}} \circ_{\bar{\nu}_{p+q}} \circ \alpha_{p+q} \circ \iota_{p, q}$ defines the desired morphism. This completes the proof.

Theorem 5.6. Let $\left(G_{i}, \varepsilon_{i}, \beta_{i}\right)$ for $i=1,2$, be cotriples on multiplicative fibred categories $\left(\mathfrak{X}_{i}, \mathfrak{B}, P_{\imath}\right)$. Then a cotriple $\left(G_{1} \Delta G_{2}, \varepsilon_{1} \Delta \varepsilon_{2}\right.$, $\left.\beta_{1} \Delta \beta_{2}\right)$ is defined on the fibred product $\left(\mathfrak{X}_{1} \Delta \mathfrak{X}_{2}, \mathfrak{B}, P_{1} \Delta P_{2}\right.$ ), which is also multiplicative. Assume that there exist natural transformations $\mu_{i}$ : $G_{i} \otimes G_{i} \rightarrow G_{i} \otimes$ satisfying $\left(\varepsilon_{i} \otimes\right) \circ \mu_{i}=\varepsilon_{i} \otimes \varepsilon_{i}$, where $\otimes: \mathfrak{x}_{i} \otimes \mathfrak{x}_{i} \rightarrow \mathfrak{X}_{i}$. Let $(1, \theta):\left(\mathfrak{X}_{1} \Delta \mathfrak{x}_{2}, \mathfrak{B}, P_{1} \Delta P_{2}\right) \rightarrow(\mathfrak{Y}, \mathfrak{A}, Q)$ be a covariant (contravariant) functor of the fibred product into a multiplicative abelian fibred category $(\mathfrak{V}, \mathfrak{X}, Q)$ such that a natural transformation $\nu: \otimes \circ(T, T) \rightarrow T \circ$ $(\otimes, \otimes)$ is given, where $\widehat{\otimes}: \mathfrak{V} \times \mathfrak{Y} \rightarrow \mathfrak{Y}$. Then there exists an external product in the cotriple homology (cohomology) $H_{*}\left(T\left(\boldsymbol{G}_{1} \Delta \mathbb{G}_{2}\right)\right.$ ).

The proof is similar to that of Theorem 5.5 and hence is omitted.

\section{Examples}

6.1 Torsion. Let $K$ be a commutative ring with unity. Let $\mathfrak{A}$ be the category of all $K$-algebras with algebra homomorphisms. Let $* \mathfrak{M}$ be the category with objects $(\Lambda, M)$ where $\Lambda$ is in $\mathfrak{A}$ and $M$ is a left $\Lambda$-module with module structure given by ${ }_{m} \varphi: \Lambda \otimes M \rightarrow M$ and morphisms $(\alpha, f):(\Lambda, M) \rightarrow\left(\Lambda^{\prime}, M^{\prime}\right)$ where ' $\alpha: \Lambda \rightarrow \Lambda^{\prime}$ and $f: M \rightarrow M^{\prime}$ is a $K$ homomorphism such that the diagram 


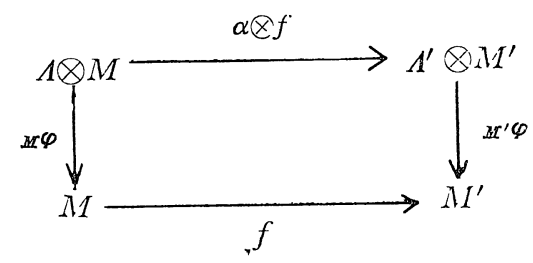

is commutative. $Q: * \mathfrak{M} \rightarrow \mathfrak{A}$ is defined by projection on the first coordinate. Then $(* \mathfrak{M}, \mathfrak{A}, Q)$ is a tensored fibred category.

Let ${ }_{\Lambda} G_{2}(\Lambda, M)=(\Lambda, \Lambda \otimes M), \quad{ }_{\Lambda} G_{2}(1, f)=(1,1 \otimes f), \quad{ }_{\Lambda} \varepsilon_{2}(\Lambda, M)=(1$, $\left.{ }_{M} \varphi\right)$ and ${ }_{\Lambda} \Delta_{2}(\Lambda, M)=(1,1 \otimes \rho \otimes 1)$ where $\rho: K \rightarrow \Lambda$ is the unit of $\Lambda$. Then $\left({ }_{1} G_{2},{ }_{1} \varepsilon_{2},{ }_{1} \Delta_{2}\right)$ is a cotriple on ${ }_{1} \mathfrak{M}=Q^{-1}(\Lambda)$ and we obtain a cotriple $\left(G_{2}, \varepsilon_{2}, \Delta_{2}\right)$ on $(* \mathfrak{M}, \mathfrak{A}, Q)$. Let $\left(\mathfrak{M}_{*}, \mathfrak{N}, P\right)$ be the category of pairs $(M, \Lambda)$ where $M$ is a right $\Lambda$-module and obtain, as for left $\Lambda$-modules, a cotriple $\left(G_{1}, \varepsilon_{1}, \Delta_{1}\right)$ of the tensored fibred category $\left(\mathfrak{M}_{*}, \mathfrak{A}, P\right)$. Let $\mu_{i}: G_{i} \otimes G_{i} \rightarrow G_{i} \otimes$ be defined by the twisting isomorphism for $i=1,2$.

Let $T: \mathfrak{M}_{*} \Delta_{*} \mathfrak{M} \rightarrow A b$ be given by $\left.T(M, \Lambda),(\Lambda, N)\right)=M \otimes{ }_{\Lambda} N$. The natural transformation $\nu$ is given by the diagram

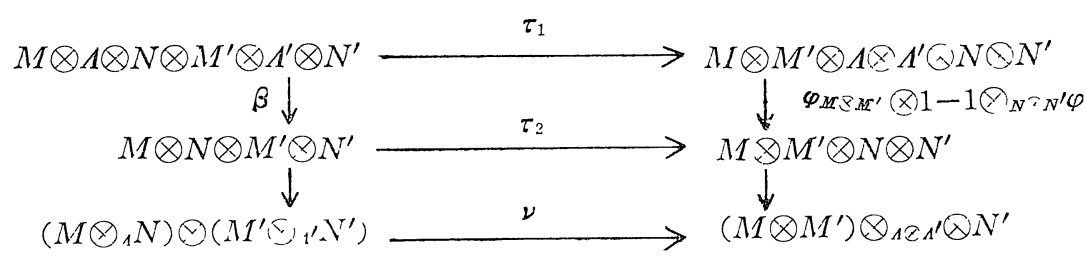

where $\tau_{1}, \tau_{2}$ are twisting isomorphisms and $\beta=\left[\left(\varphi_{M} \otimes 1-1 \otimes_{N} \varphi\right) \otimes \varphi_{M^{\prime}} \otimes 1\right]$ $+\left[1 \otimes_{N} \varphi \otimes\left(\varphi_{M^{\prime}} \otimes 1-1 \otimes_{N^{\prime} \varphi}\right]\right.$.

6.2 Cotorsion. A detailed discussion of this example is given in [1]. Let $K$ be a commutative ring with unity. Let $\mathfrak{A}$ be the category of all $K$-coalgebras and let $* \mathfrak{M}$ be the category with objects $(\Lambda$, $M$ ) where $\Lambda$ is in $\mathfrak{A}$ and $M$ is a left $\Lambda$-comodule, [5], and morphisms $(\alpha, f):(\Lambda, M) \rightarrow\left(\Lambda^{\prime}, M^{\prime}\right)$, where $\alpha: \Lambda \rightarrow \Lambda^{\prime}$ is in $\mathfrak{A}$ and $f: M \rightarrow M^{\prime}$ is a $K$-homomorphism such that the diagram 


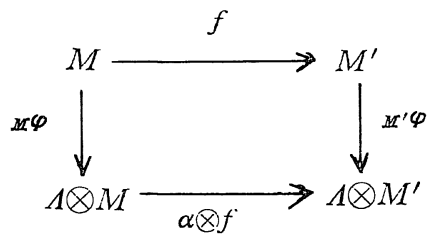

is commutative. $Q:{ }^{*} \mathfrak{M} \rightarrow \mathfrak{A}$ is defined by projection. Then $(* \mathfrak{M}, \mathfrak{A}, Q)$ is a tensored fibred category of push out type. Define a triple $\left({ }_{1} F_{2},{ }_{{ }_{1} \varepsilon_{2}}\right.$, $\left.{ }_{\Lambda} \omega_{2}\right)$ on ${ }^{\Lambda} \mathfrak{M}=Q^{-1}(\Lambda)$ by ${ }_{\Lambda} F_{2}(\Lambda, M)=(\Lambda, \Lambda \otimes M),{ }_{{ }_{1} \epsilon_{2}}(\Lambda, M)=\left(1,{ }_{M} \varphi\right)$ and ${ }_{A} \omega_{2}(\Lambda, M)=(1,1 \otimes \epsilon \otimes 1)$ where $\varepsilon: \Lambda \rightarrow K$ is the counit of $\Lambda$. Then we have a triple $\left(F_{2}, \iota_{2}, \omega_{2}\right)$ on the fibred category (*M্M, $\left.\mathfrak{N}, P\right)$. Similarly, we define $\left(\mathfrak{M}^{*}, \mathfrak{A}, P\right)$ of right $\Lambda$-comodules for any $\Lambda$ in $\mathfrak{A}$ and a triple $\left(F_{1}, \iota_{1}, \omega_{1}\right)$ on the tensored fibred category $\left(\mathfrak{M}^{*}, \mathfrak{A}, P\right)$. Let $\mu_{i}$ be defined by the twisting isomorphism and let $T: \mathfrak{M}^{*} \Delta^{*} \mathfrak{M} \rightarrow A b$ be given by $T((M, \Lambda),(\Lambda, N))=M \square N$. The natural transformation $\nu$ is given by the diagram

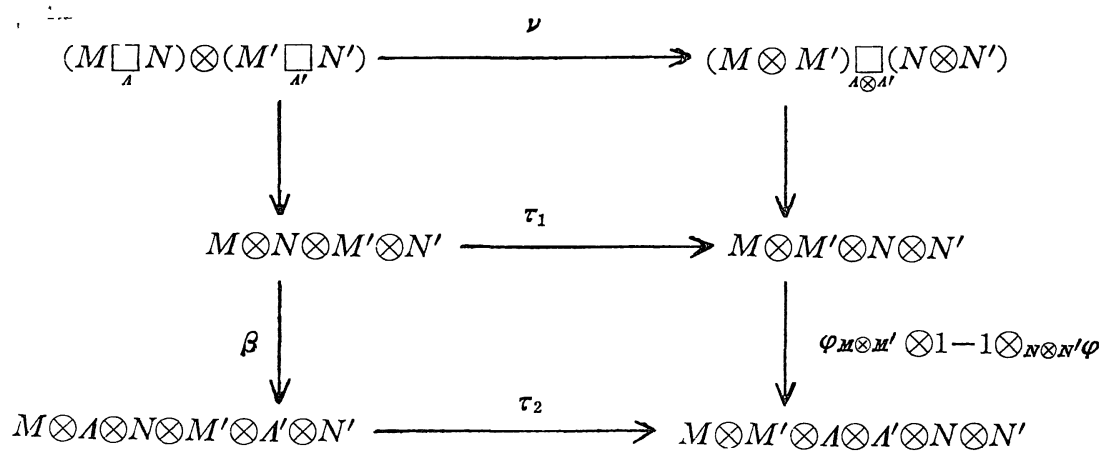

where $\tau_{1}, \tau_{2}$ are twisting isomorphisms and $\beta=\left[\left(\varphi_{M} \otimes 1-1 \otimes_{N} \varphi\right) \otimes \varphi_{M^{\prime}} \otimes 1\right]$ $+\left[1 \otimes_{N} \varphi \otimes\left(\varphi_{M^{\prime}} \otimes 1-1 \otimes_{N^{\prime}} \varphi\right)\right]$.

6.3 Extension. Let $(* \mathfrak{M}, \mathfrak{A}, Q)$ be the tensored fibred category defined in 6.1 and let $\left(G_{1}, \epsilon_{1}, \Delta_{1}\right)$ be the cotriple defined on $(* \mathfrak{M}, \mathfrak{A}$, $Q)$. $\mu_{1}$ is the twisting isomorphism; $\mu_{1}: G_{1} \circ \otimes \rightarrow \otimes\left(G_{1}, G_{1}\right)$. Let ( ${ }^{\sharp}$, $\widehat{\mathfrak{A}}, P)$ be the tensored fibred category of push out type defined in section 1 and $(F, \iota, \omega)$ is the triple given there. Let $\left(G_{2}, \varepsilon_{2}, \Delta_{2}\right)$ be the

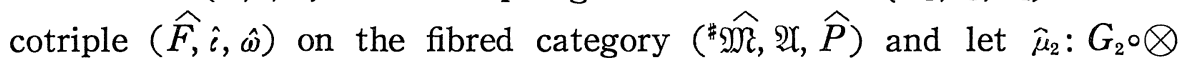


$\rightarrow \otimes\left(G_{2}, G_{2}\right)$ be given by $\mu_{2}: \operatorname{Hom}_{K}(\Lambda, N) \otimes \operatorname{Hom}_{K}\left(\Lambda^{\prime}, N^{\prime}\right) \rightarrow \operatorname{Hom}_{K}(\Lambda \otimes$ $\left.\Lambda^{\prime}, N \otimes N^{\prime}\right)$. We then consider $T: * \mathfrak{M} \Delta^{\sharp} \hat{\mathfrak{M}} \rightarrow A b$ defined by $T((\Lambda, M)$, $(\Lambda, \widehat{N}))=\operatorname{Hom}_{\Lambda}(M, N)$. Let $\nu: \operatorname{Hom}_{1}(M, N) \otimes \operatorname{Hom}_{A^{\prime}}\left(M^{\prime}, N^{\prime}\right) \rightarrow \operatorname{Hom}_{\Lambda \varangle A^{\prime}}$ $\left(M \otimes M^{\prime}, N \otimes N^{\prime}\right)$ be defined by $\nu(\xi \otimes \beta)=\xi \otimes \beta$.

One can verify that ${ }_{A}^{* M M}=P^{-1}(\Lambda)$ is isomorphic to $A M M=Q^{-1}(\Lambda)$. Therefore, by considering complementary classes, [2], we obtain the wedge product of Ext.

6.4 Coextension. For the definition and properties of Coextension see [1]. It is also shown there that a Yoneda product exists for Coext and this product induces an algebra structure on Coext. In this paragraph we consider all $K$-coalgebras to be projective of finite type.

Let $\left({ }^{*} \mathfrak{M}, \mathfrak{N}, P\right)$ be the tensored fibred category of push out type defined in 6.2 and $(F, \iota, \omega)$ the triple on $(* \mathfrak{M}, \mathfrak{A}, P)$. Then $(\widehat{F}, \hat{\iota}, \hat{\omega})$ is a cotriple on $(\widehat{*} \mathfrak{M}, \widehat{\mathscr{A}}, \widehat{P})$. Let $\mathfrak{M}^{\sharp}$ denote all ordered pairs $(\widehat{A}, M)$ where $\hat{A}$ is in $\widehat{\mathscr{A}}$, $\widehat{\mathscr{A}}$ is the dual category of $\mathfrak{A}$, and $M$ is a $\Lambda$-contramodule, [2]. Morphisms are ordered pairs $(\hat{\alpha}, f):(\widehat{\Lambda}, M) \rightarrow\left(\widehat{\Lambda^{\prime}}, M^{\prime}\right)$ where $\hat{\alpha}: \widehat{\Lambda} \rightarrow \widehat{\Lambda}^{\prime}$ and $f: M \rightarrow M^{\prime}$ is a $K$-homomorphism such that the diagram

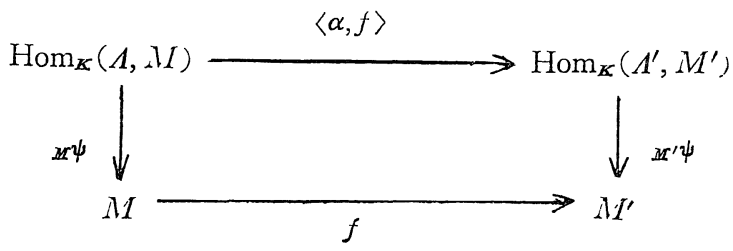

is commutative, where $\langle\alpha, f\rangle(\xi)=f \circ \xi^{\circ} \alpha$. Define $Q: \mathfrak{M}^{\sharp} \rightarrow \widehat{\mathscr{N}}$ by projecinto on the first coordinate, then $\left(\mathfrak{M}^{\sharp}, \widehat{\mathfrak{A}}, Q\right)$ is a tensored fibred category.

For each $\hat{\Lambda}$ in $\widehat{\mathscr{A}}$ let ${ }_{\Lambda} G(\widehat{\Lambda}, M)=\left(\widehat{\Lambda}, \operatorname{Hom}_{K}(\Lambda, M)\right),{ }_{\Lambda} \varepsilon(\Lambda, M)=(1$, $\left.{ }_{m} \psi\right)$ and ${ }_{\Lambda}(\Lambda, M)=\left(1,(1 \otimes c)^{*}\right)$, where $c: \Lambda \rightarrow K$ is the counit of $\Lambda$. Then $\left({ }_{1} G,{ }_{A} \varepsilon,{ }_{A} \Delta\right)$ is a cotriple on the fibre ${ }^{\wedge} \mathfrak{M R}^{\sharp}=Q^{-1}(\widehat{\Lambda})$ and a cotriple $(G, \varepsilon, \Delta)$ is obtained on the fibred category $\left(\mathfrak{M}^{\sharp}, \widehat{\mathfrak{A}}, Q\right)$. 
Let $\mu_{1}: G \circ \otimes \rightarrow \otimes(G, G)$ be given by the isomorphism $\operatorname{Hom}_{K}\left(\Lambda_{1} \otimes\right.$ $\left.\Lambda_{2}, M_{1} \otimes M_{2}\right) \cong \Lambda_{1}^{*} \otimes \Lambda_{2}^{*} \otimes M_{1} \otimes M_{2} \cong \Lambda_{1}^{*} \otimes M_{1} \otimes \Lambda_{2}^{*} \otimes M_{2} \cong \operatorname{Hom}_{K}\left(\Lambda_{1}, M_{1}\right) \otimes$ $\operatorname{Hom}_{K}\left(\Lambda_{2}, M_{2}\right)$ and let $\mu_{2}: \widehat{F} \circ \bigotimes \rightarrow \bigotimes(\widehat{F}, \widehat{F})$ be given by the twisting isomorphism. Define $T: \mathfrak{M}^{\sharp} \Delta^{*} \widehat{M} \rightarrow A b$ by $T((\widehat{A}, M),(\widehat{\Lambda}, \widehat{N}))=$ $\operatorname{Hom}_{A}(M, N)$. Then $T$ is a contravariant additive functor. Let $\nu: \otimes$ $(T, T) \rightarrow T \circ(\otimes, \otimes)$ be given by $\nu(\xi \otimes \beta)=\xi \otimes \beta$ for $\xi: M \rightarrow N$ and $\beta$ : $M^{\prime} \rightarrow N^{\prime}$.

Since the coalgebras considered are projective of finite type, $\mathfrak{M R}^{\#} \cong$ *M্R, [2], and by the consideration of complementary classes one obtains an external product for Coext.

\section{References}

[1] Brenneman, F. S., Derived functors in relative homological algebra, Doctoral dissertation, Oklahoma State University, $196^{\circ} 7$.

[2] Eilenberg, S. and J. C. Moore, Foundations of relative homological algebra, Mem. Amer. Math. Soc. 55 (1965).

[3] , Adjoint functors and triples, Illinois J. Math. 9 (1965), 381-398.

[4] Gray, J. W., Fibred and cofibred categories, Proceedings of the Conference on Categorical Algebra, La Jolla, Calif. (1966), 21-83.

[5] Milnor, J. W. and J. C. Moore, On the structure of Hopf algebras, Ann. of Math. 81 (1965), 211-264.

[6] Shimada, N., H. Uehara and F. S. Brenneman, Cotriple cohomology in relative homological algebra, submitted. 